\title{
Sediment-entraining suspension clouds: a model of powder-snow avalanches
}

\author{
By M. RASTELLO ${ }^{1} \dagger$ AND E. J. HOPFINGER ${ }^{2}$ \\ ${ }^{1}$ CEMAGREF, 2 rue de la Papeterie, B.P. 76, 38402 Saint Martin d'Hères cedex, France \\ ${ }^{2}$ LEGI-CNRS, B.P. 53, 38041 Grenoble Cedex, France
}

(Received 28 October 2002 and in revised form 26 February 2004)

A dense cloud model of avalanches is presented which includes large density difference effects as well as sediment entrainment along the path of the cloud. This model demonstrates the importance of sediment entrainment in the evolution of the front velocity. Without sediment entrainment the cloud first accelerates and then decelerates, a behaviour known from previous studies of cloud or thermal motions. With sediment entrainment the cloud is mostly in an accelerating state. The closure coefficients in the model concerning the cloud shape and air entrainment are obtained from laboratory experiments. These coefficients can be considered generic in the Boussinesq limit. A correction for inertial effects which need to be taken into account when applied to large density difference clouds such as avalanches, is proposed. An expression for the sediment entrainment coefficient is derived, taking into account the flow parameters and the sediment layer properties. The model predictions are in good agreement with recent measurement of the front velocity of a powder-snow avalanche. A presentation, in terms of dimensionless variables, of avalanche and laboratory Boussinesq cloud velocities shows clearly the similarities and differences between the two.

\section{Introduction}

Powder-snow avalanches are large-scale, finite-volume-release turbidity currents, or more specifically, large-scale suspension clouds, occurring on inclines of initial slope angle larger than about $30^{\circ}$. In order to give a feeling of the size of powder-snow avalanches an image is shown in figure 1. The size can be estimated by comparison with the trees in front of the avalanche. These clouds can reach $100 \mathrm{~m}$ in height and front velocities of the order of $100 \mathrm{~m} \mathrm{~s}^{-1}$. Similar velocities are observed in pyroclastic flows (Calder et al. 1999). For an avalanche to reach such large velocities, it must be constantly accelerating and the effective drag must be very small. Hopfinger (1983), in his review, compiled data which show a fairly close correlation of the avalanche velocity with the snow cover depth. The bulk density of a powder-snow avalanche is about ten times that of the surrounding air which raises additional difficulties because the Boussinesq approximation can no longer be used. In addition, the bulk density and stratification are due to the suspended snow particles and, generally, energy is required to keep these particles in suspension. Further discussions of the essential features of these flows and their relation with gravity currents in general are given by Simpson (1997) and by Hutter (1996). 


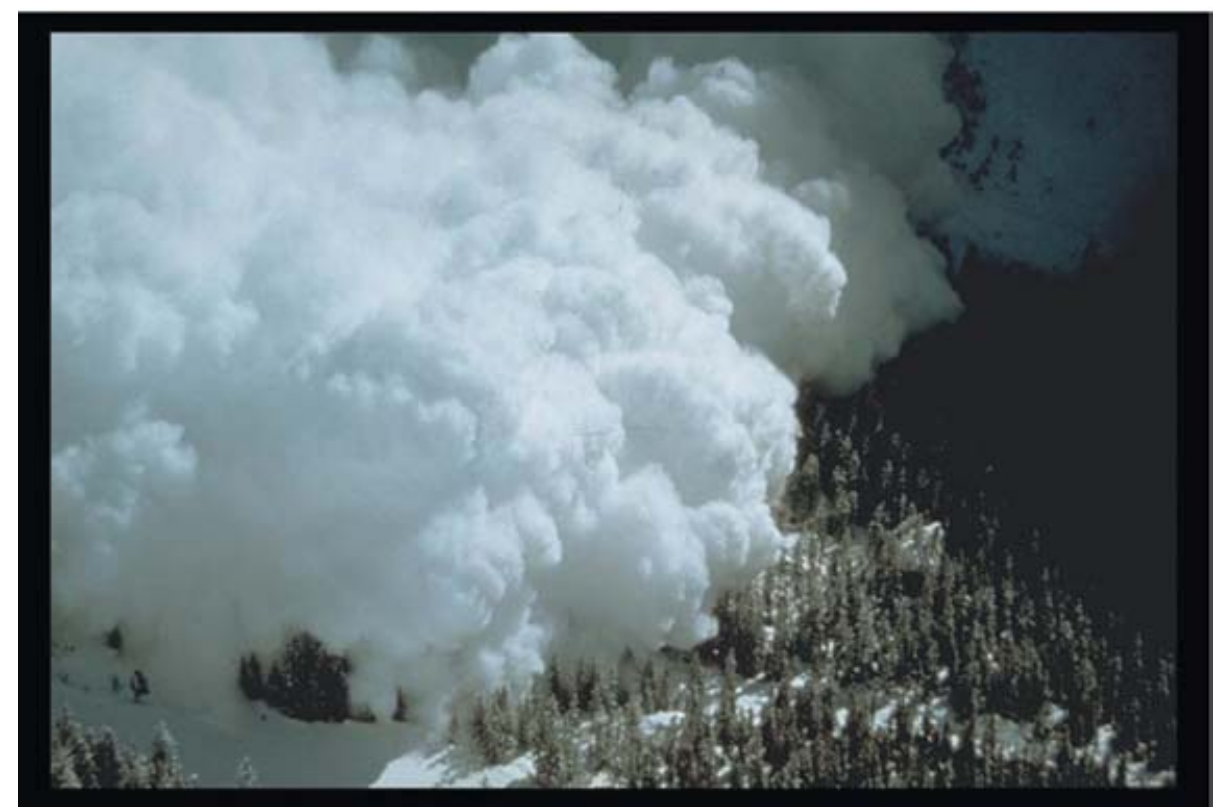

FIGURE 1. The front of a powder-snow avalanche (photo courtesy of C. Vion).

Tochon-Danguy \& Hopfinger (1975) extended the depth-integrated turbulent gravity current model of Ellison \& Turner (1959) (further developed in Turner 1972), to non-Boussinesq gravity currents representing avalanches when it can be assumed that the snow particle fall velocity is much smaller than the turbulent velocity. An expression for the velocity was obtained in the steady state limit, neglecting snow entrainment and using an air entrainment coefficient at the upper edge obtained from experiments with Boussinesq currents. Powder-snow avalanches are mostly finite-volume-release events, forming a cloud or thermal on an incline. When snow entrainment is neglected the bulk density of the avalanche decreases rapidly along the path and the maximum velocity that can be reached is considerably less than what is usually reported. Indeed, when snow entrainment is not taken into account the velocity, after an initial acceleration phase, decreases with distance in a way similar to Boussinesq thermals on inclines (Beghin, Hopfinger \& Britter 1981). Hopfinger \& Tochon-Danguy (1977) proposed a simple model of snow entrainment which will be developed further in the present paper in a way similar to Beghin \& Brugnot (1983). Fukushima \& Parker (1990) also extended the cloud model of Beghin et al. (1981) by allowing for large density differences and by taking into account sediment entrainment or deposition, using criteria taken from open-channel flow hydraulics. These criteria are in the same form as those used for continuous turbidity currents by Parker, Fukushima \& Pantin (1986). The main interest was in determining the conditions for sediment entrainment (igniting flow conditions) rather than deposition. These conditions of course depend critically on the sediment entrainment model used as well as on the air entrainment coefficient. These authors also examined the kinetic energy equation (giving a four-equation model) and concluded that for avalanche conditions the energy required to keep the sediment in suspension is a negligible part of the turbulent energy production. A three-equation model (continuity, mass and momentum conservation) is, therefore, adequate as long as we are interested in mean flow quantities. 
Recent measurements of an avalanche front velocity by geo-referenced images and radar in the Sion valley (Dufour, Gruber \& Ammann 2001), clearly demonstrate a continuous increase of the front velocity with distance and the complete incorporation of the snow cover of thickness 1 to $1.5 \mathrm{~m}$. Another important result is that the maximum velocity measured by radar inside the avalanche behind the leading edge exceeds the front velocity by about $30 \%$ to $40 \%$. The images taken (personal communication, F. Dufour) indicate a finite length of the avalanche and a height of about 50 to $80 \mathrm{~m}$ at a down-slope distance of about $1500 \mathrm{~m}$. The slope angle ranged from nearly $45^{\circ}$ at the start to about $20^{\circ}$ at the lower end.

These results motivated us to reconsider the avalanche models and in particular the three-equation model developed previously by including in the model sediment (snow) entrainment and large density difference effects. Laboratory experiments with Boussinesq clouds moving over a sediment layer were conducted in order to determine more precisely the air entrainment coefficient and the effect sediment entrainment has on the dynamics of the cloud moving down steep slopes. A complete analytical solution for the velocity is presented together with expressions for the closure parameters of sediment and air (ambient fluid) entrainment determined from the laboratory experiments performed.

In $\S 2$ we present the cloud model which allows for snow or sediment entrainment and large density differences between the flow and the ambient fluid. A general solution for the cloud velocity is obtained and expressions for the asymptotic velocity limits are given. The closure coefficients in the model are discussed, also giving an expression for the sediment entrainment coefficient. In $\S 3$ the laboratory experiments are described and the results (concerning the different parameters of the model) are presented in $\S 4$. In $\S 5$ the cloud velocity and conditions of sediment entrainment are analysed. Section 6 is concerned with the comparison between the Swiss avalanche, the model and the laboratory results. Conclusions and further discussion are presented in $\S 7$.

\section{The dense cloud model}

\subsection{General formulation of the model}

We consider a finite volume or suspension cloud flow on an incline, as suggested by Hopfinger \& Tochon-Danguy (1977) and sketched in figure 2.

For a two-dimensional cloud that entrains air and sediment, the momentum, continuity and mass conservation equations are

$$
\begin{gathered}
\frac{\mathrm{d}\left(\rho+k_{v} \rho_{a}\right) A U}{\mathrm{~d} t}=\Delta \rho A g \sin \theta-C_{f} \rho_{b} U^{2} L, \\
\frac{\mathrm{d} A}{\mathrm{~d} t}=E_{c} \frac{\sqrt{S_{1}}}{S_{2}} U P=E_{c} U \sqrt{A}
\end{gathered}
$$

with $A=S_{1} k H^{2}$ and $P=S_{2} \sqrt{H L}$ or alternatively

$$
\begin{gathered}
\frac{\mathrm{d} H}{\mathrm{~d} t}=\frac{E_{c} U}{2 \sqrt{S_{1} k}}, \\
\frac{\mathrm{d} \Delta \rho A}{\mathrm{~d} t}=\beta \Delta \rho_{s} h_{s} U=E_{s} \Delta \rho_{s} L U,
\end{gathered}
$$

where $S_{1}$ and $S_{2}$ are shape factors (kept constant in the present model), $k(\theta)=L / H$ is the aspect ratio (ratio of length to height of the cloud), $A$ the surface area and $P$ the 


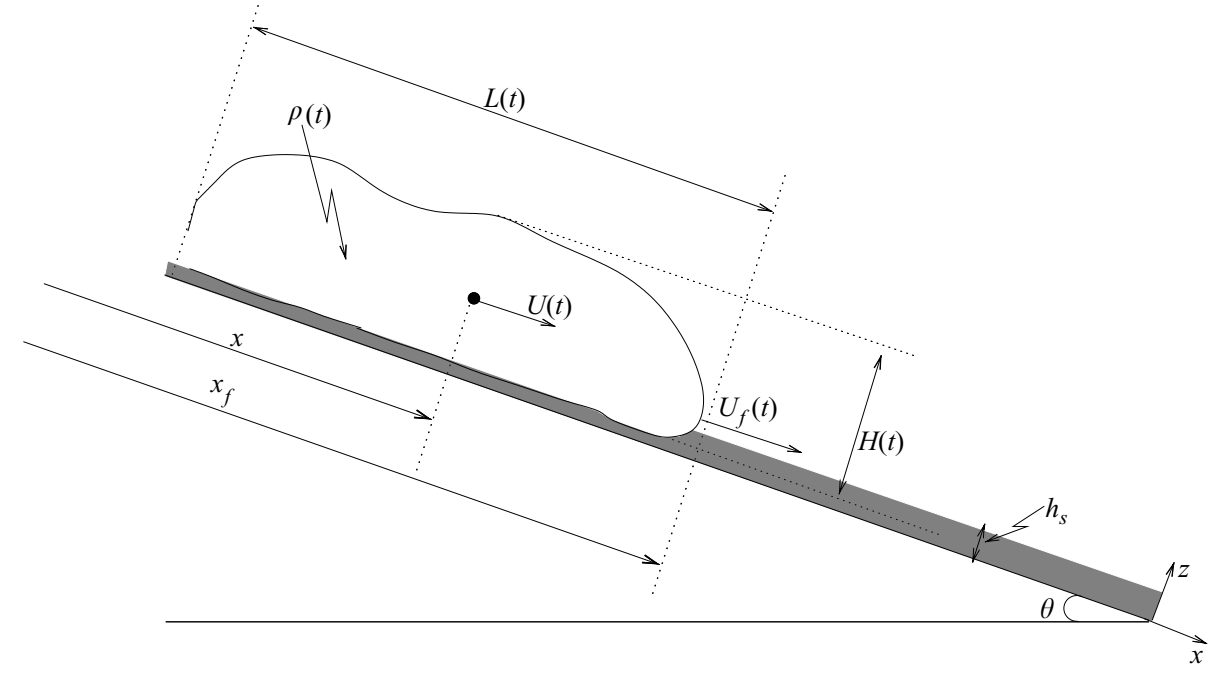

FIGURE 2. Sketch of a cloud or thermal on an incline.

length of the interface between the cloud and the ambient fluid. The other variables are: $\rho=\rho_{p} C+\rho_{a}(1-C)$ the mixture density, $\Delta \rho=\rho-\rho_{a}$ the density difference between the cloud and the ambient fluid of density $\rho_{a}, \rho_{p}$ the particle density, $C$ the volume concentration of particles, $\rho_{b}$ the density of the cloud near the sediment layer (bottom of the cloud), $k_{v}$ the added mass coefficient, $C_{f}$ the friction coefficient on the bottom, $\beta$ the fraction of the sediment layer of thickness $h_{s}$ and density $\rho_{s}$ entrained by the cloud along the path, $E_{c}$ the entrainment coefficient of ambient fluid and $E_{s}$ the sediment entrainment coefficient.

In a real avalanche, the boundary is very rough so that $C_{f} \approx 10^{-2}$ in the bottom friction term on the right-hand side of (2.1). Even with this value of $C_{f}$, the bottom friction term is on average an order of magnitude smaller than the driving force term. To make things less complicated we neglect this friction term in the following analytical development and then determine its effect by numerical integration of the above set of equations. Fukushima \& Parker (1990) also included an interfacial friction between the cloud and the ambient fluid in (2.1). Such an interfacial friction is negligible compared with bottom friction (because mean velocity gradients are much weaker near the fluid interface) and certainly has no effect on the flow. Thus, the main force balance is between the buoyancy force and the momentum transferred to the entrained ambient fluid and also to the entrained sediment.

In (2.1) to (2.3) the derivative with respect to time can be replaced by spatial derivatives using $U=\mathrm{d} x / \mathrm{d} t$. Integration of these equations, keeping $E_{c}$ and $\beta$ constant, gives

$$
\begin{gathered}
A=\left(\sqrt{A_{0}}+\frac{E_{c}\left(x-x_{0}\right)}{2}\right)^{2}, \\
H=H_{0}+\frac{E_{c}\left(x-x_{0}\right)}{2 \sqrt{S_{1} k}}, \\
\Delta \rho A=\left(\Delta \rho_{0} A_{0}-\beta \Delta \rho_{s} h_{s} x_{0}\right)+\beta \Delta \rho_{s} h_{s} x, \\
U=\frac{\sqrt{f(x)+V_{0}^{2}-f\left(x_{0}\right)}}{M+B x+N x^{2}},
\end{gathered}
$$




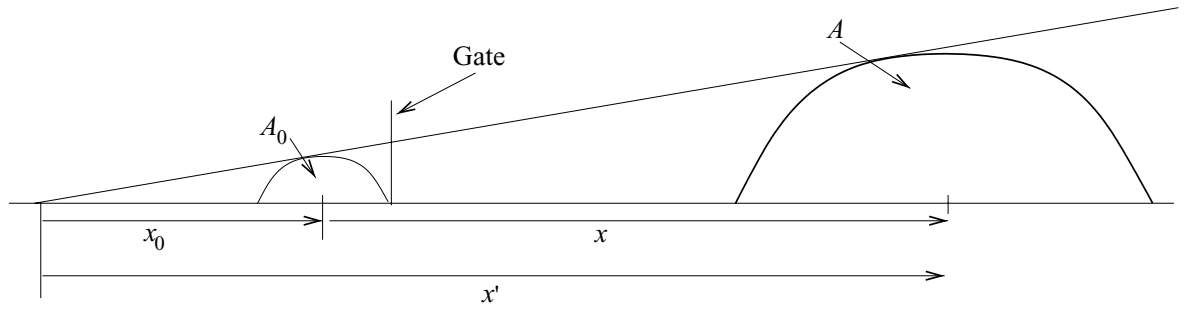

FIGURE 3. Definition of initial distance from the virtual origin $x_{0}$ and of the positions $x$ and $x^{\prime}$.

where

$$
\begin{gathered}
V_{0}=\left(M+B x_{0}+N x_{0}^{2}\right) U\left(x_{0}\right), \\
f(x)=2 K M x+(K B+G M) x^{2}+\frac{2}{3}(K N+G B) x^{3}+\frac{1}{2} G N x^{4},
\end{gathered}
$$

with

$$
\begin{gathered}
B=\beta \Delta \rho_{s} h_{s}+\left(1+k_{v}\right) \rho_{a}\left(E_{c} \sqrt{A_{0}}-x_{0} \frac{1}{2} E_{c}^{2}\right), \\
M=\left(1+k_{v}\right) \rho_{a}\left(\sqrt{A_{0}}-\frac{1}{2} E_{c} x_{0}\right)^{2}+\Delta \rho_{0} A_{0}-\beta \Delta \rho_{s} h_{s} x_{0}, \\
N=\left(1+k_{v}\right) \rho_{a} \frac{1}{4} E_{c}^{2}, \\
K=\left(\Delta \rho_{0} A_{0}-\beta \Delta \rho_{s} h_{s} x_{0}\right) g \sin \theta, \\
G=\beta \Delta \rho_{s} h_{s} g \sin \theta .
\end{gathered}
$$

In the above theory the position $x$ (we should call it $x^{\prime}$ ) is measured from a virtual origin $x^{\prime}=x+x_{0}\left(x_{0}=2 \sqrt{A_{0}} / E_{c}\right)$ in a way similar to that usually used for plumes and jets for instance (see figure 3). At $x^{\prime}=x_{0}$ the cloud has a finite volume per unit width $A_{0}$ and without loss of generality we can set $x_{0}=0$ and measure $x$ from the physical origin, but taking into account the finite values of $A_{0}$ and possibly $U_{0}$ at $x=0$. An analytical solution can be obtained when it is assumed, in addition to neglecting bottom friction, that $x_{0}=0$ and $U_{0}=0$ at $x=0$ so that $V_{0}=0$ and $f(0)=0$. This solution gives for the velocity:

$$
U=\frac{\sqrt{2 K M x+(K B+G M) x^{2}+\frac{2}{3}(K N+G B) x^{3}+\frac{1}{2} G N x^{4}}}{M+B x+N x^{2}} .
$$

Although this expression for $U$ is similar to the one obtained by Beghin \& Brugnot (1983) we thought it necessary to reformulate the problem in a consistent way.

When comparing the model results with laboratory experiments or real avalanches, it is more useful to work with the variables related to the front of the flow (coordinate $x_{f}$ and velocity $\left.U_{f}\right)$ rather than with the variables $(x, U)$ linked to the mass centre. The relation between the position of the front $x_{f}$ and the position of the mass centre $x$ is $x_{f}=x+L / 2$. The relation which gives the front velocity $U_{f}$ as a function of the mass centre velocity $U$ is $U_{f}=U\left(1+\frac{1}{2} \mathrm{~d} L / \mathrm{d} x\right)$.

\subsection{Examination of asymptotic limits}

In order to have a better appreciation of the flow behaviour it is of interest to determine the asymptotic velocity limits in the near ( $x$ 'small') and far field ( $x$ 'large') respectively. For large-density-difference clouds (avalanches), when $x$ is small, the dominant term in the numerator of (2.8) is $2 K M x$ and in the denominator $M$ 
dominates. The expression for $U$ is therefore

$$
U \approx \sqrt{2 g x \sin \theta} .
$$

This asymptotic expression for entirely free fall is valid for $x \ll \sqrt{M / N}$ in the absence of sediment entrainment and for $x \ll M / B$ when entrainment of sediment is important $\left(\beta h_{s} \approx 1 \mathrm{~m}\right)$. The order of magnitude of $\sqrt{M / N}$ is $1400 \mathrm{~m}, M / B \approx 195 \mathrm{~m}$, for an avalanche such as that of the Sion valley (Swiss avalanche) described in $\S 6$ which is typical of large-scale powder-snow or mixed-snow avalanches $\left(A_{0} \approx 200 \mathrm{~m}^{2}\right.$, $\left.\Delta \rho_{0} \approx \rho_{s} \approx 150 \mathrm{~kg} \mathrm{~m}^{-3}, \beta h_{s} \approx 1 \mathrm{~m}\right)$. For Boussinesq, buoyancy-conserving clouds, we obtain when $x \ll M / B$

$$
U \approx \sqrt{\frac{\Delta \rho_{0} 2 g x \sin \theta}{\left(1+k_{v}\right) \rho_{a}}} .
$$

The distance over which this regime is observed depends on the initial conditions and is for our laboratory clouds (see $\S 4) x \ll M / B \approx 0.3 \mathrm{~m}$.

In the other limit, of large $x$, the expressions are quite different with and without entrainment of sediment. For the avalanche without snow entrainment we obtain

$$
U \approx \sqrt{\frac{8 \Delta \rho_{0} A_{0} g \sin \theta}{3\left(1+k_{v}\right) \rho_{a} E_{c}^{2} x}}
$$

and this expression is valid when $x \gg \sqrt{3 M / N}(\sqrt{3 M / N} \approx 2500 \mathrm{~m}$ for conditions corresponding to the Swiss avalanche). This limit velocity is the same for buoyancyconserving, Boussinesq clouds, but in this case it occurs for $x \gg 3 B / 2 N \approx 1.7 \mathrm{~m}$ for the present laboratory clouds. In the experiments of Beghin et al. (1981) the nearand far-field limits are about half the values indicated here (for the same slope angle).

With snow entrainment the far-field velocity limit is obtained when $x \gg 4 B /$ $3 N \approx 9000 \mathrm{~m}$ (for the Swiss avalanche) and is

$$
U \approx \sqrt{\frac{2 \Delta \rho_{s} \beta h_{s} g \sin \theta}{\left(1+k_{v}\right) \rho_{a} E_{c}^{2}}} .
$$

It is interesting to note that in the far-field limit the flow velocity $U$ is proportional to $\sqrt{h_{s}}$. This result corresponds to observations (Hopfinger 1983). Another interesting remark is that the distance over which this regime is valid increases like $\sqrt{\beta h_{s}}$. Indeed, the more sediment (snow) is entrained, the farther downstream the entrainment of ambient fluid is able to balance the driving buoyancy force.

\subsection{Choice of the closure parameters}

Any model requires as input knowledge of the topography (at least the slope angle $\theta$ ) and the initial conditions, which are here the initial volume per unit width of dense fluid released $A_{0}$, the initial excess density $\Delta \rho_{0}$, the initial velocity $U_{0}$ and the excess density $\Delta \rho_{s}$ and depth $h_{s}$ of the sediment layer.

The model developed above also requires closure parameters which are essentially $E_{c}$ and $\beta$ or $E_{s}$. These have to be determined either from experiments or, possibly, from numerical simulations which at present do not exist. The other parameters, which are $k, k_{v}, C_{f}$ and the shape factors, affect the flow to a much lesser extent. The shape factors are close to the values of a half-elliptical body $\left(S_{1} \approx 0.80\right.$ and $\left.S_{2} \approx 2.4\right)$ and the term in $C_{f}$ in $(2.1)$ can be neglected as long as the slopes are steep $\left(\theta>5^{\circ}\right.$ for Boussinesq clouds somewhat larger for non-Boussinesq clouds). The added mass coefficient is taken constant, equal to 0.5 , which is slightly less than the value $k_{v}=2 / k$ 
proposed by Fukushima \& Parker (1990). When the cloud density increases its effect decreases.

\subsubsection{Entrainment coefficient $E_{c}$ and geometric parameters}

The air (ambient fluid) entrainment coefficient $E_{c}$, related with the spatial growth rate through $(2.2 b)$, is a generic quantity in the Boussinesq limit. Beghin et al. (1981) determined $\mathrm{d} H / \mathrm{d} x_{f}$ from visual observations of the height of the cloud at different downstream positions. The experimental points show a large scatter but indicate clearly a linear dependence on slope angle. The spatial growth was approximated by $\mathrm{d} H / \mathrm{d} x_{f} \approx 3.6 \times 10^{-3} \theta+0.04$ taking into account the fine sand suspension clouds. The shape factors were shown to also be functions of the slope angle.

In the laboratory experiments presented in $\S 3$ and $\S 4$, the coefficient $E_{c}$ was directly determined from the evolution of the integral of the cloud volume per unit width, also giving the shape factors. The value of $\mathrm{d} H / \mathrm{d} x_{f}$ was determined from the evolution of the height of the cloud and also indirectly from the value of $E_{c}$ using the shape factors. All these geometric values determined experimentally are used as characteristic values in the Boussinesq limit.

The large density difference between the flow and the ambient fluid (a ratio of order 10 in powder-snow avalanches) will certainly affect the spatial growth rate by the inertial effect, in a way similar to what is observed in large-density-difference shear layers (Brown \& Roshko 1974; Dimotakis 1986). The experiments by Brown \& Roshko (1974) showed that when the higher velocity flow is the larger density fluid, the spatial growth rate is reduced, mainly because the advection velocity of the coherent structures is increased. Maslowe \& Kelly (1971) showed that the inertial effect on the spatial growth rate of disturbances in a heterogeneous shear layer is the opposite of the effect on the temporal growth rate. The flow is stabilized with respect to spatially growing disturbances when the heavier fluid has the higher velocity whereas the flow tends to be more unstable with respect to the temporal growth rate. The turbulent, heterogeneous shear layer has a spatial growth rate qualitatively consistent with these linear stability results (Brown \& Roshko 1974).

A sketch of an avalanche or dense cloud in a reference frame moving with $U_{f}$ is shown in figure 4 . The important point to note is that the maximum velocity near the ground or bed, behind the front, referred to as $U_{b}$, is considerably larger than the front velocity. Dufour et al. (2001) reported maximum velocities inside the avalanche about $30 \%$ to $40 \%$ larger than the front velocity. In the Boussinesq limit the velocity behind the front can be larger than the front velocity by a factor of about two (Hopfinger \& Tochon-Danguy 1977; Britter \& Linden 1980). The growth rate of the avalanche height is about half that which would be predicted for Boussinesq laboratory clouds and the ratio of length to height (factor $k$ in equation $(2.2 b)$ ) is about two times larger. The entrainment coefficient $E_{c}$, which is needed in the expression for the cloud velocity, can then be determined from equation $(2.2 b)$ assuming similarity of the shape which is suggested by observations. One possibility is to use these values as closure parameters in the cloud model. It is, however, of interest to seek an expression for the change in $\mathrm{d} H / \mathrm{d} x_{f}$ and $E_{c}$ as a function of the density ratio. A change in entrainment by inertial effects will mainly affect the growth in the height and it can therefore be assumed that the growth of the length is unaffected.

The general expression for the spatial growth rate (growth of a free shear flow) is

$$
\frac{\mathrm{d} H}{\mathrm{~d} x}=\alpha(\theta) \frac{\Delta U}{U_{c}},
$$




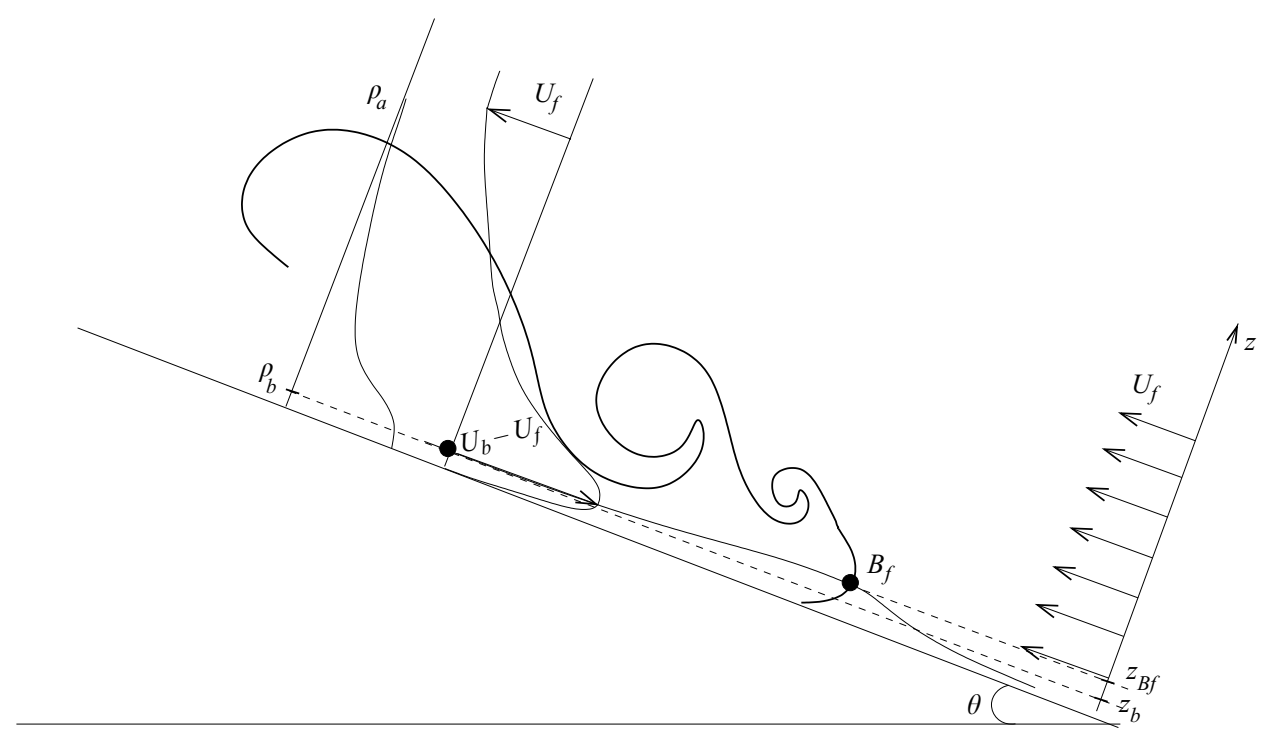

FIGURE 4. Sketch of the cloud in the reference frame moving with velocity $U_{f}$.

where $\Delta U=U_{b}-U_{a}=U_{b}$ because $U_{a}=0$. The velocity $U_{b}$ is the maximum velocity behind the head close to the bottom boundary where the mean flow streamlines are nearly parallel. It is most likely that the streamline corresponding to $U_{b}$ stagnates at the nose at point $B_{f}$ (see figure 4). The large structures move with the cloud but their convection speed is $U_{c} \leqslant U_{f}$ where $U_{f}$ is the velocity of the cloud front. The difference between the two is due to the growth in length and is approximately the same in Boussinesq and non-Boussinesq clouds. We can, therefore, replace $U_{c}$ by $U_{f}$ in equation (2.13) since the proportionality coefficient can be included in the coefficient $\alpha$. The main task is then to obtain an expression for $U_{b}$ in terms of $U_{f}$.

The flow is turbulent but the mean flow is quasi-steady $(H / U \ll x / U)$. We can, therefore, write the Bernoulli equation along a streamline which stagnates at point $B_{f}$ in the form

$$
p_{b}+\frac{1}{2} \rho_{b}\left(U_{b}-U_{f}\right)^{2}-g \cos \theta\left(\rho_{b}-\rho_{a}\right)\left(z_{B f}-z_{b}\right)=p_{a}+\frac{1}{2} \rho_{a} U_{f}^{2}
$$

or equivalently

$$
\frac{2\left(p_{b}-p_{a}\right)}{\rho_{a} U_{f}^{2}}+\frac{\rho_{b}}{\rho_{a}} \frac{\left(U_{b}-U_{f}\right)^{2}}{U_{f}^{2}}-2 R i_{\Delta z}=1,
$$

where $R i_{\Delta z}=g \cos \theta\left(\rho_{b}-\rho_{a}\right)\left(z_{B f}-z_{b}\right) /\left(\rho_{a} U_{f}^{2}\right)$. In deriving equation (2.14) it is assumed that the density $\rho_{b}$ remains constant along the streamline, which is strictly speaking not the case because of mixing. It is well known, however, that mixing in stratified fluids is inefficient (Strang \& Fernando 2001) so that this assumption is close to reality. The same assumption was made by Dimotakis (1986) in the case of a turbulent, inhomogeneous shear layer and there is good agreement with experiments (the experiments by Brown \& Roshko 1974). In the inhomogeneous shear layer of Brown \& Roshko (1974) the Richardson number is very small and gravity terms are negligible but mixing is then even larger than in a flow where only gravity effects are important. A small density variation along the streamline could be included in the derivation of equation $(2.14 a)$ but there is no reason to do this. The term $R i_{\Delta z} \ll 1$ because $\left(z_{B f}-z_{b}\right) \ll H$ and can be neglected. The hydrostatic pressure term, 
$2\left(p_{b}-p_{a}\right) / \rho_{a} U_{f}^{2}=2 \Delta \rho g \cos \theta\left(H-z_{b}\right) / \rho_{a} U_{f}^{2}$ is also relatively small (evaluated to be about 0.3 ) because $\Delta \rho$ in the outer part of a cloud (Hadano 1981) or even in a gravity current (Turner 1972) is much less than $\left(\rho_{b}-\rho_{a}\right)$. Note that this is consistent with the fact that on steep slopes the main driving force is the down-slope gravitational force and the hydrostatic pressure is principally reponsible for the increase in the length of the cloud. This is different from a gravity flow on a horizontal boundary where the hydrostatic pressure is the driving force and is, therefore, the dominant term.

As a first approximation we can write $\left(1-2\left(p_{b}-p_{a}\right) /\left(\rho_{a} U_{f}^{2}\right)\right)^{1 / 2} \approx 1$ so that

$$
U_{b} \approx U_{f}\left(1+\sqrt{\frac{\rho_{a}}{\rho_{b}}}\right) .
$$

The inertial effect on the spatial growth rate of the cloud is therefore

$$
\frac{\mathrm{d} H}{\mathrm{~d} x}=\alpha(\theta)\left(1+\sqrt{\frac{\rho_{a}}{\rho_{b}}}\right) .
$$

Applying this relation to both Boussinesq and non-Boussinesq clouds we obtain the following inertial correction for the growth rate $\uparrow$ :

$$
\frac{\mathrm{d} H}{\mathrm{~d} x_{N B}} \approx \frac{\mathrm{d} H}{\mathrm{~d} x_{B}} \frac{1+\sqrt{\rho_{a} / \rho_{b}}}{2}
$$

where subscripts $B$ and $N B$ refer to Boussinesq and non-Boussinesq respectively.

The inertial effect on the variation of the length of the cloud is difficult to evaluate. It is to be expected that the large density difference would tend to increase rather than decrease the evolution in length of the cloud. In the absence of any clear concept we assume that the evolution in length is not appreciably changed by inertial effects and use the length obtained in the Boussinesq limit. Therefore,

$$
k_{N B} \approx k_{B} \frac{2}{1+\sqrt{\rho_{a} / \rho_{b}}} .
$$

This gives

$$
E_{c N B} \approx E_{c B}\left(\frac{1+\sqrt{\rho_{a} / \rho_{b}}}{2}\right)^{1 / 2} .
$$

Equation (2.19) indicates that $E_{c}$ has a much weaker dependence on the density ratio than the spatial growth rate in height. This assumes of course that $k$ is affected by the density ratio but not the length.

It should be mentioned here that in previous publications (Hopfinger 1983; Baines \& Hopfinger 1984) it was argued that the spatial growth rate is increased by inertial effects when the faster moving fluid has a larger density. These arguments, based on dimensional analysis, did not consider the change in advection velocity of the coherent structures. The inertial correction given by (2.15), (2.17) and (2.19) is qualitatively in agreement with observations of powder-snow avalanches.

\subsubsection{Sediment entrainment coefficient}

The coefficient $\beta=E_{s} L / h_{s}$ has to be related to the flow parameters. The approach taken by Fukushima \& Parker (1990) was to extend directly results obtained for

$\dagger$ Implicit in the above reasoning is that the interfacial instability develops rapidly so that the reduction in spatial growth rate, when the density ratio is large, is due to an increase in the convection velocity of the coherent structures. 
continuous turbidity currents to turbidity clouds. Such an approach is doubtful because the cloud structure is quite different from that of a developed turbidity current. At the present state of knowledge it is safer to use basic energy arguments in a way similar to Celik \& Rodi $(1984,1991)$ for instance. The right-hand side of the mass conservation equation (2.3) can be written in the form

$$
\frac{\mathrm{d} \Delta \rho A}{\mathrm{~d} t} \leqslant\left(\rho_{b}-\rho_{a}\right) w_{s} \cos \theta\left(C_{b m}-C_{b}\right) L
$$

where $C_{b}$ is the near-bed concentration, $C_{b m}$ the maximum near-bed concentration the flow is able to carry and $w_{s}$ the particle settling velocity. The inequality in (2.20) arises because $C_{b m} w_{s} \cos \theta$ is the maximum sediment entrainment rate. This equation expresses the rate of change of potential energy (if multiplied by $g$ ) due to sediment entrainment or deposition, depending on the sign of $\left(C_{b m}-C_{b}\right)$. The problem remains to determine the maximum mean concentration, $C_{m}$, and the ratios $\kappa_{1}=C_{b m} / C_{m}$ and $\kappa_{2}=C_{b} / C$. The excess density variation with distance from the bed has only been measured in the head of a saline gravity current (Hadano 1981) where $\Delta \rho_{b} / \Delta \rho \approx 2$. In the absence of measurements in suspension clouds it is reasonable to assume that $\kappa_{1} \approx \kappa_{2} \approx 3,\left(C_{b m}-C_{b}\right) \approx 3\left(C_{m}-C\right)$. The value of $C_{m}$ is determined from energy arguments assuming that the part of the turbulent kinetic energy necessary to keep the particles in suspension is a small, constant portion $\gamma$ of the turbulent kinetic energy production per unit mass $\overline{u w} \partial U / \partial z$ :

$$
\gamma \rho \overline{u w} \frac{\partial U}{\partial z}=\left(\rho_{p}-\rho_{a}\right) C_{m} g w_{s} \cos \theta .
$$

For the mean flow gradient we can take $2 \alpha_{1} U_{f} / H$ with $\alpha_{1} \approx 2$ in Boussinesq and 1.5 in non-Boussinesq clouds. The Reynolds stress is $\overline{u w} \approx 0.5 u^{\prime} w^{\prime}$ with $u^{\prime} \approx 0.3 \alpha_{1} U_{f}$ and $w^{\prime}=K(\theta) U_{f}$. The angle dependence (or Richardson number dependence) of $K$ is the effect of stratification on the vertical turbulent velocity component. This turbulent velocity is directly related with the entrainment velocity $w_{a}$ at the fluid interface and is $w^{\prime}=2 w_{a}=2 E_{c f} S_{1}^{1 / 2} U_{f} / S_{2}$, giving $K \approx 2 E_{c f} S_{1}^{1 / 2} / S_{2}$. The expression for $C_{m}$ is therefore

$$
C_{m}=\gamma \frac{0.3 \rho \alpha_{1}^{2} K U_{f}^{3}}{\left(\rho_{p}-\rho_{a}\right) g H w_{s} \cos \theta} .
$$

The value of $\gamma$ will be determined from the experiments with sediment entraining clouds (see $\S 4$ and $\S 5$ ) and applied to the Swiss avalanche in $\S 6$. Note that the condition is that $\gamma \ll 1$. The expression for $\beta=E_{s} L / h_{s}$ is obtained from (2.3) and (2.20):

$$
\beta \leqslant \frac{3\left(\rho_{p}-\rho_{a}\right) w_{s} \cos \theta\left(C_{m}-C\right) L}{\left(\rho_{s}-\rho_{a}\right) U_{f} h_{s}} .
$$

A necessary condition for self-maintenance of a suspension in a turbidity current of depth-averaged mean velocity $\bar{U}$ suggested by Bagnold (1962) is $w_{s} \cos \theta / \bar{U} \sin \theta \leqslant 1$. Stacey \& Bowen (1988) suggest $w_{s} \cos \theta / \bar{U} \sin \theta \leqslant 0.01$ to 0.1 for self-maintenance of a turbidity current on a rough wall. The values of $w_{s} \cos \theta / U \sin \theta$ quoted for an avalanche range from 0.1 to 0.01 , of the same order as the values suggested by Stacey \& Bowen (1988).

A necessary condition for sediment motion is that the shear stress is larger than a critical value $\tau_{c}$. On a flat bed the value of $\tau_{c r i t}$ for incipient particle motion is obtained from the Shields diagram for open-channel flow hydraulics (see for instance Graf 1984) giving the critical shear stress or, more precisely, the critical Shields number 
$\Theta_{\text {crit }}=\tau_{\text {crit }} /\left(\rho_{p}-\rho\right) g d$ as a function of the particle Reynolds number $R e_{*}=u^{*} d / \nu$, where $u^{*}=(\tau / \rho)^{1 / 2}$ is the friction velocity and $d$ the particle diameter. The friction velocity is $u^{* 2}=C_{f} U_{b}^{2}$. On a slope the critical Shields number $\Theta_{c}=\Theta_{c r i t} \sin (\alpha-\theta) / \sin \alpha$ (Hogg, Huppert \& Dade 1997), where $\alpha$ is the angle of repose $\left(\alpha=20^{\circ}\right.$ to $40^{\circ}$ depending on the sediment). Therefore on steep slopes $\Theta_{c} \approx 0$. The critical shear stress for the beginning of particle suspension $\tau_{c s}$ is less well-established. Existing hydraulics data show that on a flat bed $\tau_{c s}$ or $\Theta_{c s}=\tau_{c s} /\left(\rho_{p}-\rho\right) g d$ is nearly independent of $R e_{*}$ when $R e_{*}$ is large and increases at small $R e_{*}$. On a slope we replace $g$ by $g \cos \theta$ to calculate $\Theta_{c s}$. Celik \& Rodi (1984) analysed existing experimental data on sediment suspension which suggest

$$
\Theta_{c s}=\left\{\begin{array}{lll}
0.25 R e_{* T} / R e_{*} & \text { when } & R e_{*} \leqslant R e_{* T} \\
0.25 & \text { when } & R e_{*} \geqslant R e_{* T}
\end{array}\right.
$$

(These critical values are determined for water. We use the same conditions for air (avalanches) which might not be entirely correct.) For the transition value $R e_{* T}$ Celik $\&$ Rodi (1984) propose 0.6. Some experiments even suggest $R e_{* T} \approx 20$. It seems that most data show an increase in $\Theta_{c s}$ when $R e_{*}<2$.

The rate of suspension is a function of $\Theta$ and by analogy with bed-load transport we propose

$$
q_{s}=K_{s} w_{s} \cos \theta\left(\Theta-\Theta_{c s}\right)^{3 / 2} \leqslant C_{b m} w_{s} \cos \theta
$$

where $K_{s}$ is a constant. The $3 / 2$ power giving a $U^{3}$ velocity dependence is consistent with (2.22) when $\Theta \gg \Theta_{c s}\left(q_{s}=C_{b m} w_{s} \cos \theta\right.$ when $\left.\Theta \gg \Theta_{c s}\right)$.

\section{Laboratory experiments with sediment-entraining clouds}

In order to understand the physics of powder-snow avalanches and to determine their growth rate and capacity of sediment entrainment, it is essential to perform laboratory experiments with Boussinesq clouds on inclines which are limiting cases of real avalanches. A good theoretical model should then include these limiting Boussinesq cases and allow the extrapolation of laboratory results to real avalanches. The aim of the present experiments was to determine the conditions of sediment entrainment (determine $\beta$ and hence $\gamma$ and $C_{m}$ ) and measure the spatial growth rate of the clouds to obtain a Boussinesq reference value for saline clouds as well as suspension clouds. It was also of interest to obtain a qualitative indication of how sediment is entrained into the cloud.

\subsection{The experimental setup}

The experimental installation is shown schematically in figure 5. The transparent water channel is $2 \mathrm{~m}$ long, $30 \mathrm{~cm}$ wide and $40 \mathrm{~cm}$ deep. This channel is immersed in a large water tank $2 \mathrm{~m}$ deep. The slope angle of the experimental test channel can be varied from $0^{\circ}$ to $50^{\circ}$. In the experiments reported, the angle was varied between $30^{\circ}$ and $45^{\circ}$. The dense fluid was prepared in a rectangular section $20 \mathrm{~cm} \times 20 \mathrm{~cm} \times 30 \mathrm{~cm}$ located at the upper end of the test channel and separated from the rest by a sluice 


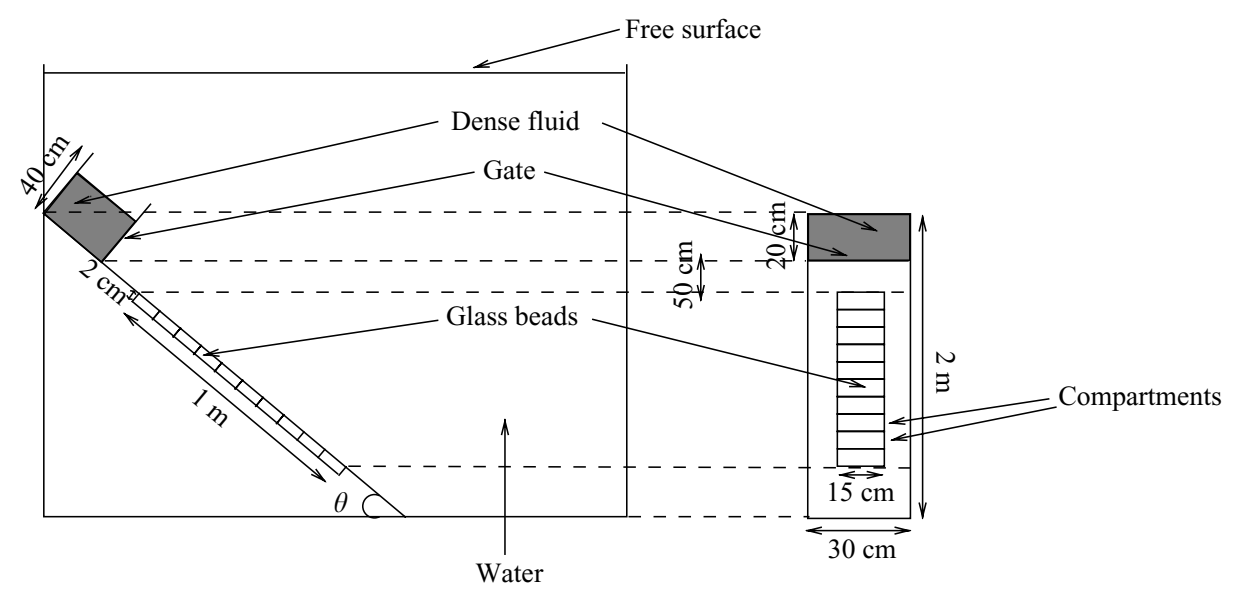

FIGURE 5. Schematic representation of the experimental tank.

gate. The dense fluid consisted either of saltwater of initial volume $A_{0}=0.013 \mathrm{~m}^{2}$ and of variable initial density $\left(\Delta \rho_{0} / \rho_{a} \approx 10 \%\right)$ or of a particle suspension of $A_{0}=0.04 \mathrm{~m}^{2}$ and $\Delta \rho_{0} / \rho_{a} \approx 0.4 \%$. The suspended were sawdust of density $\rho_{p} \approx 1060 \mathrm{~kg} \mathrm{~m}^{-3}$ when wet and $500 \mu \mathrm{m}$ mean diameter. The shape of these particles is irregular in a way similar to suspended snow particles. Their settling velocity in water is $w_{s} \approx 0.8 \mathrm{~cm} \mathrm{~s}^{-1}$ giving a ratio $w_{s}$ to front velocity of about 0.08 . This is comparable with the ratio in an avalanche. The Stokes numbers are also similar; in both cases $S t<0.1$.

Simulating the snow cover along the bottom is a difficult task mainly because of the slope but also because of the very porous nature of the snow cover. In the case of the suspension clouds a rough bottom was established by gluing sawdust particles to the floor. Then it was possible to deposit a 1 to $2 \mathrm{~mm}$ thick sawdust sediment layer before starting the experiment. In the experiments with saltwater clouds a $2 \mathrm{~cm}$ thick sediment layer of glass beads was carefully deposited in a compartmented reservoir $1 \mathrm{~m}$ long and $15 \mathrm{~cm}$ wide, starting $50 \mathrm{~cm}$ downstream of the gate. The partitions in this reservoir, necessary to retain the sediment, introduce some roughness which is thought to be of little importance since the entrainment of ambient fluid dominates the dynamics. The glass beads were of $90 \mu \mathrm{m}$ microns mean diameter, giving a settling velocity of $w_{s} \approx 0.6 \mathrm{~cm} \mathrm{~s}^{-1}$. With a salt solution it is easier than with a suspension to produce clouds of larger velocities and to keep initial perturbations at a low level. This is due to the larger initial density difference that can be used. Typical front velocities, when reaching the sediment layer, were $U_{f}=25 \mathrm{~cm} \mathrm{~s}^{-1}$, giving $w_{s} / U_{f} \approx 0.03$. This is again of the same order as the values quoted for powder-snow avalanches. The advantage of salt solution clouds is that these are transparent which allow visualization of the sediment entrainment process.

\subsection{Measurement techniques}

The spatial growth rate was determined from side view images obtained with a video camera. This growth rate is defined as the change with distance $x_{f}$ of the visual height. The entrainment coefficient $E_{c}$ was determined from measurements of the change with distance $x_{f}$ of the surface area of the cloud determined from the side view images, using $(2.2 a)$. The saline clouds were made visible by adding a dye to the dense fluid volume released, whereas in the case of the suspension clouds the particles 


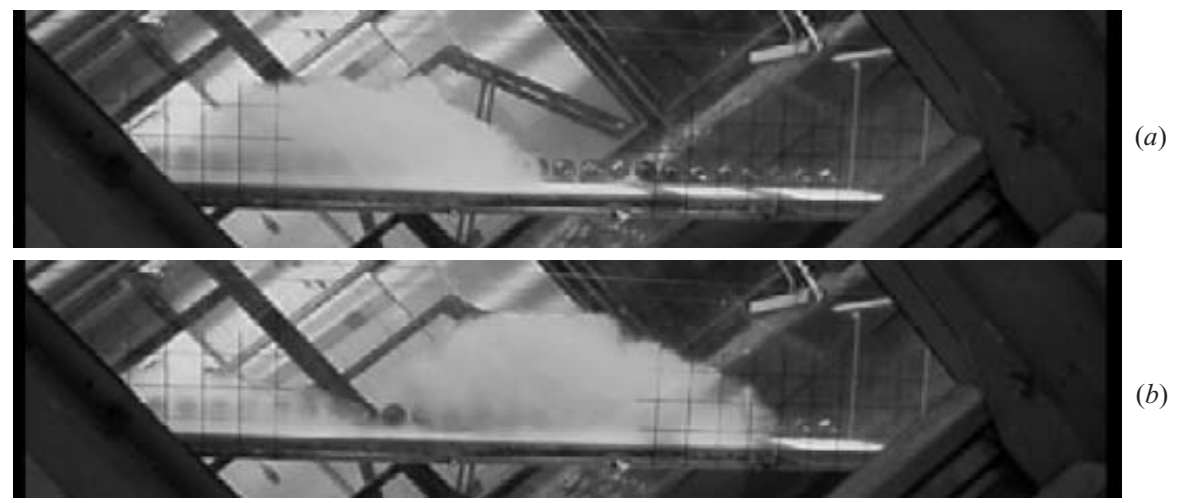

FIGURE 6. Side view of a saline cloud moving down a smooth slope of angle $32^{\circ}:(a)$ the front is $120 \mathrm{~cm}$ from the gate, $(b)$ the front is $155 \mathrm{~cm}$ from the gate. The grid lines on the image are a $5 \mathrm{~cm}$ mesh.

in suspension defined the cloud contour. The range over which the cloud growth was determined was $2<x_{f} / A_{0}^{1 / 2}<13$. The height and volume per unit width were measured at intervals $\Delta x \approx 4 \mathrm{~cm}$ in the case of the suspension clouds and $\Delta x \approx 20 \mathrm{~cm}$ in the saline clouds. The spread and entrainment coefficient $E_{c}$ were then determined from linear regression fitted to the data, that is respectively $H$ the height measured at the centre of the cloud and $\sqrt{A}$ as a function of $x_{f}$. A total of 5 to 10 experiments were analysed for each case.

In the case of the suspension clouds, the front velocity was measured by taking top view video images of the leading edge. In the case of the saline clouds conductivity probes were used to determine the passage of the leading edge. A total of 29 probes were positioned along the channel. The space between two successive probes was $\Delta x=5 \mathrm{~cm}$. The sensitive tip was $5 \mathrm{~cm}$ from the sidewall and $5 \mathrm{~cm}$ above the bottom. The local velocity of the front is then given by $U_{f}=\Delta x / \Delta t$ where $\Delta t$ is the time taken for the front of the flow to pass between two probes. The observed fluctuations in $U_{f}$ were as large as $\pm 30 \%$. The results of $U_{f}$ shown in figure 11 are filtered values (averaged over 4 points). The results obtained by this technique give an indication of the variability of the front velocity with respect to the mean.

\section{Experimental results}

\subsection{Shape and growth rate of the clouds}

A side view of a saline cloud moving down a slope of $32^{\circ}$ is shown in figure 6 . The growth of the cloud due to the entrainment of ambient fluid is clear from these images. In the wake of the cloud some dense fluid is left behind (see figure $6 b$ ) and moves slowly down the slope at a slower speed than the cloud. An overall view of the suspension cloud is shown in figure 7. This image shows similarities with a real avalanche and clearly indicates the variability of the leading edge. Figure 8 and figure 9 show the results concerning the growth rate and the aspect ratio. The large spread in the experimental data is of physical origin and is related with the large-eddy motions in the cloud and the lobe-and-cleft structure of the front. The suspension cloud tends to have a slightly smaller growth rate. The mean spreading rate, $\mathrm{d} H / \mathrm{d} x_{f}$, 


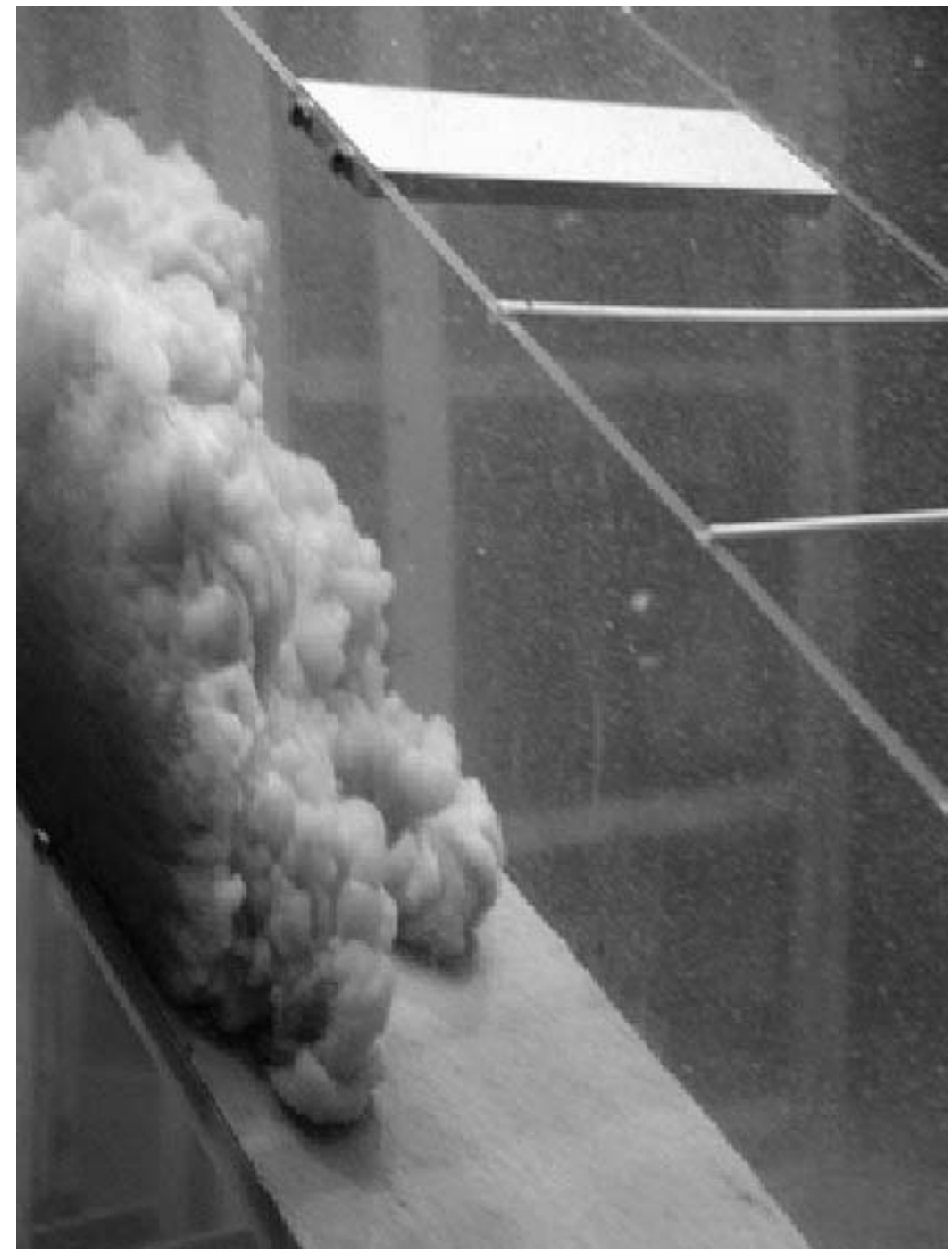

Figure 7. Frontal view of a sawdust suspension cloud on a slope of $45^{\circ}$. (Photo courtesy of C. Ancey.)

of the saline cloud lies on a straight line approximated by

$$
\frac{\mathrm{d} H}{\mathrm{~d} x_{f}}=3.6 \times 10^{-3} \theta+0.013
$$

which connects the present results with the growth of the line thermal for $\theta=90^{\circ}$ (Tsang 1971). The spread of saline clouds found by Beghin et al. (1981) is also wellapproximated by this correlation. The lower spread (by about 10\%) of the suspension clouds is attributed to the settling of the suspended particles.

As mentioned previously, the entrainment coefficient was determined directly from $E_{f c}=2\left(\mathrm{~d} \sqrt{A} / \mathrm{d} x_{f}\right)$ which is related to $E_{c}$ by $E_{f c}=E_{c}\left(1-\frac{1}{2} \mathrm{~d} L / \mathrm{d} x_{f}\right) . E_{f c}$ was also determined from $\mathrm{d} H / \mathrm{d} x_{f}$, using $(2.2 b)$ and the value of $k$. The mean values for $k=L / H, S_{1}=A / L H, S_{2}=P / \sqrt{H L}, \mathrm{~d} H / \mathrm{d} x_{f}, E_{f c}$ and $E_{c}$ are given in table 1 . The 


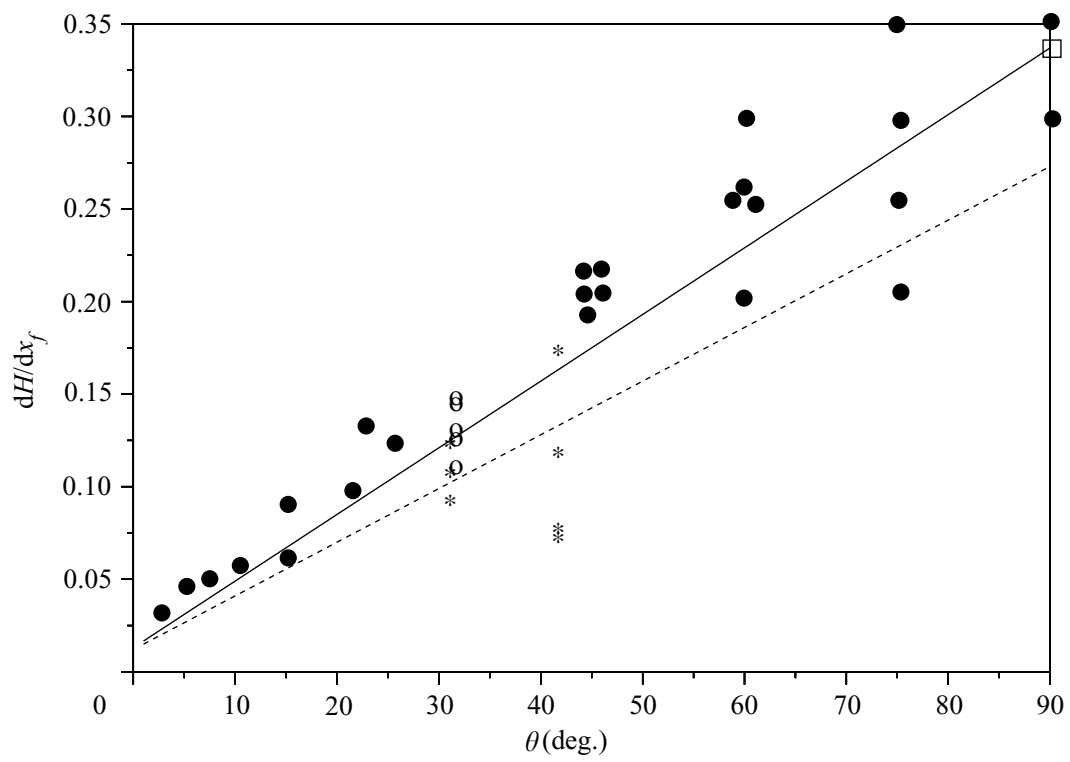

Figure 8. Spatial growth rate of the height as a function of slope angle: $\bullet$, experiments with saline clouds of Beghin et al. (1981); - - - , growth rate of a gravity current head with constant speed (Britter \& Linden 1980); o, present experiments with laboratory saline clouds; *, present experiments with sawdust suspension clouds $\left(w_{s} / U_{f} \approx 0.1\right)$; $\square$, line thermal of Tsang (1971) for $\theta=90^{\circ} ;-$, line $\mathrm{d} H / \mathrm{d} x_{f}=3.6 \times 10^{-3} \theta+0.013$.

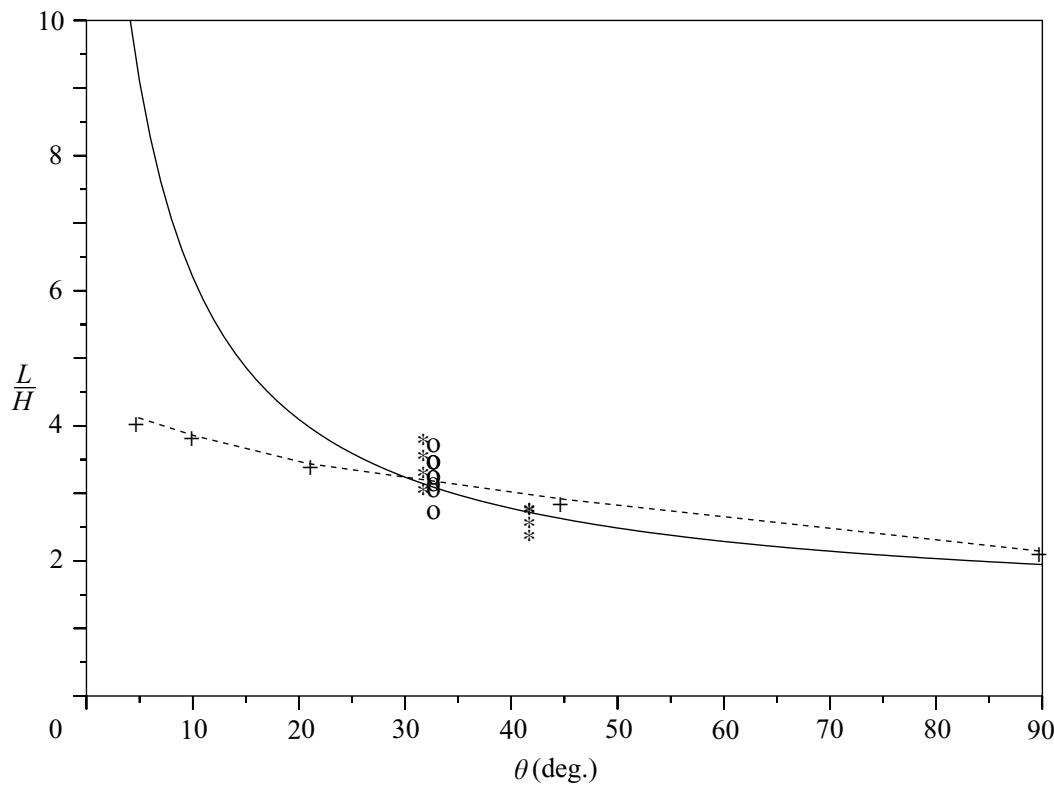

Figure 9. Aspect ratio $k=L / H$, of the saline and suspension clouds: - - values of Beghin et al. (1981) saline clouds; + - - - +, values of Britter \& Linden (1980) obtained for a gravity current head; o, present experiments with saltwater; *, present experiments with sawdust suspension clouds. 


\begin{tabular}{lccccccc}
\hline & $k$ & $S_{1}$ & $S_{2}$ & $\mathrm{~d} H / \mathrm{d} x_{f}$ & $E_{f c}$ & $E_{c}$ & $w_{s} / U_{f}$ \\
Saline cloud (DC), $\theta=32^{\circ}$ & 3.2 & 0.78 & 2.43 & $0.13 \pm 10 \%$ & $0.27 \pm 14 \%$ & $0.32 \pm 14 \%$ & 0 \\
Suspension cloud (SC), $\theta=32^{\circ}$ & 3.3 & 0.78 & 2.45 & $0.10 \pm 12 \%$ & $0.25 \pm 22 \%$ & $0.30 \pm 22 \%$ & 0.1 \\
Suspension cloud (SC), $\theta=42^{\circ}$ & 2.5 & 0.81 & 2.39 & $0.11 \pm 37 \%$ & $0.28 \pm 13 \%$ & $0.34 \pm 13 \%$ & 0.1
\end{tabular}

TABLE 1. Mean experimental values of the aspect ratio $k$, the shape factors $S_{1}$ and $S_{2}$, the spatial growth rate, the entrainment coefficients and the ratio between the settling velocity of the suspended particles and the front velocity of the cloud.
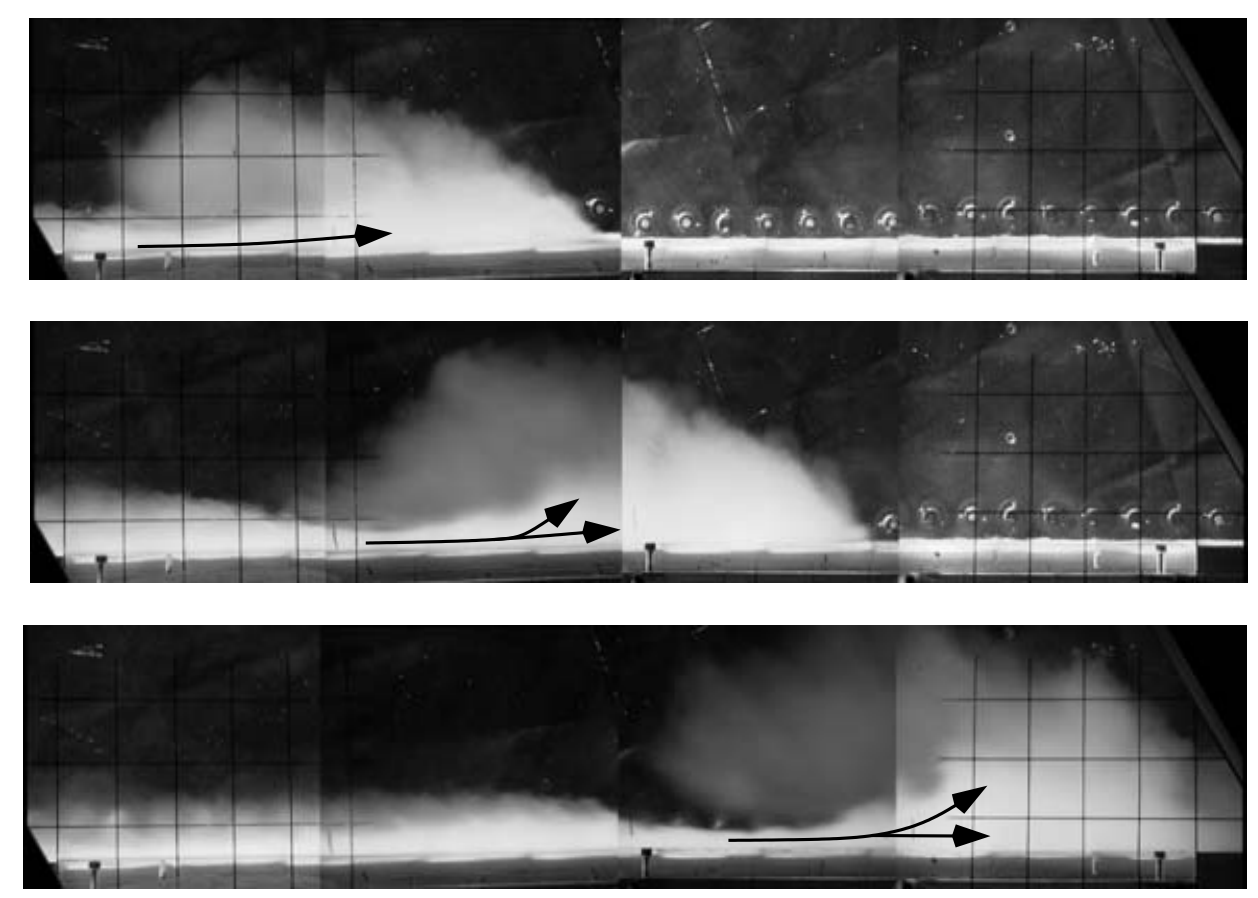

FIGURE 10. Image showing the sediment lift-up in a laboratory saltwater cloud moving over a thick layer of glass beads (white indicates high concentration of glass beads).

uncertainty of these values indicated in table 1 is not experimental error but is due to the very large variability in height due to the large structures.

\subsection{Qualitative observations of sediment entrainment}

The experiments with saltwater clouds moving over a thick layer of glass beads, deposited on the floor prior to the experiments, allowed visualization of the sediment entrainment process. This entrainment is clearly not uniform; the rate of entrainment is largest a certain distance behind the front (see the arrows in figure 10) and then the entrained sediment is partially moved toward the front because the velocity behind the front exceeds the front velocity. The sediment lift-up might be enhanced by a suction effect due to the large-eddy structures which cause relatively large negative pressures (of the order of $\rho U_{f}^{2} / 2$ ) toward the rear of the cloud (Hopfinger 1983). 


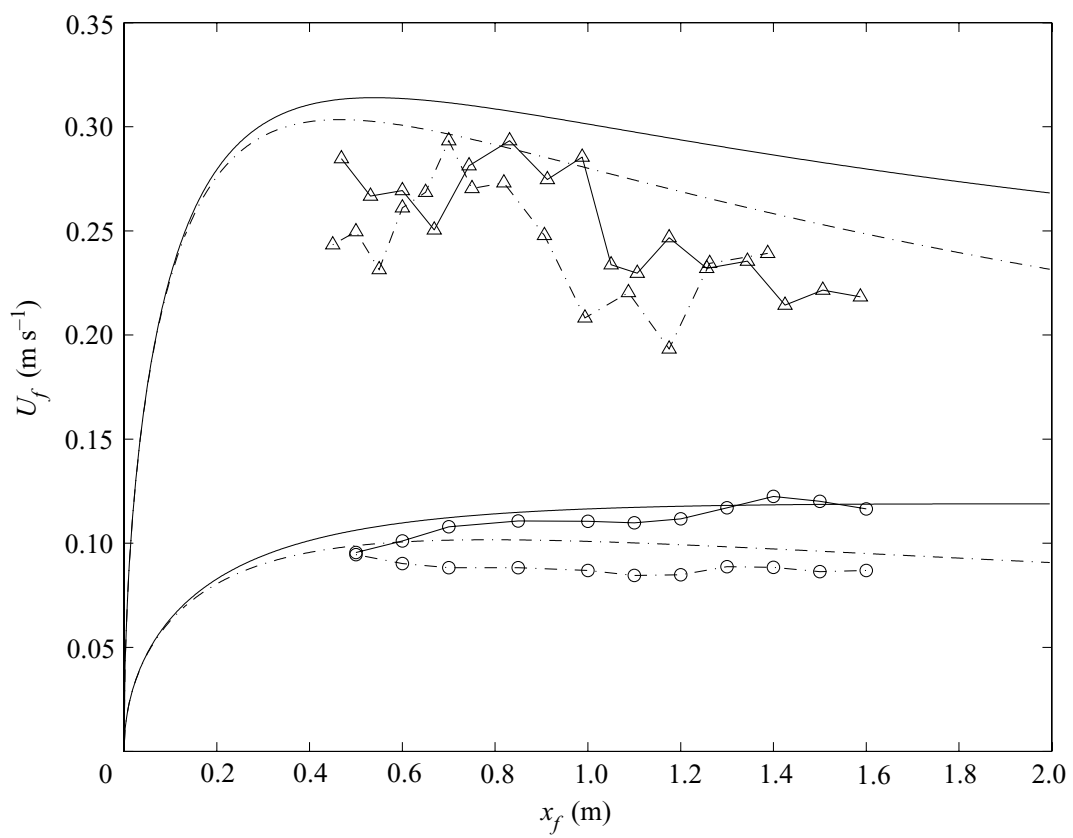

FiguRE 11. Evolution of the front velocity of the laboratory clouds as a function of downstream distance: $-\cdot-\triangle \cdot-\cdot$, measured velocity of a saline cloud moving over a smooth bottom of $\theta=32^{\circ} ;-\cdot-\cdot-\cdot$, calculated velocity with $\theta=32^{\circ}$ and $E_{c}=0.35 ;-\triangle-$, measured front velocity of a saline cloud moving over the glass beads sediment layer with $\theta=32^{\circ}$;

calculated velocity with $\theta=32^{\circ}, E_{c}=0.35, \beta=2.5 \times 10^{-2}, h_{s}=2 \mathrm{~cm} ;-\cdot-\circ \cdot-\cdot$, front velocity of a suspension cloud without sediment entrainment and $\theta=45^{\circ} ;-\circ-$, front velocity of a suspension cloud with entrainment and $\theta=45^{\circ} ;-\cdot-\cdot-$ and - (lower), are the respective model predictions with $\theta=45^{\circ}$ and $E_{c}=0.35$. The velocity in the case with entrainment was calculated with $h_{s}=0.2 \mathrm{~cm}$ and $\beta=1$.

Some picked-up sediment is also left behind the cloud in the form of a (passive) wake.

\subsection{Front velocities}

Figure 11 shows typical front velocities for saline and suspension clouds with and without sediment entrainment. The initial excess mass is $\Delta \rho_{0} A_{0}=1.3 \mathrm{~kg} \mathrm{~m}^{-1}$ in the saline clouds $\left(A_{0}=0.013 \mathrm{~m}^{2}\right)$ and $\Delta \rho_{0} A_{0}=0.16 \mathrm{~kg} \mathrm{~m}^{-1}$ in the suspension clouds $\left(A_{0}=\right.$ $0.04 \mathrm{~m}^{2}$ ). It is seen that when the clouds move over the sawdust layer or the layer or glass beads, the velocity is larger. This is an indication of sediment entrainment, confirmed by visual observations as seen in figure 10 for the saline cloud. The suspension cloud entrained the whole layer of $2 \mathrm{~mm}$ thickness. The saline cloud entrained only a small fraction of the deposited layer.

In the case of the sawdust clouds the front velocity was averaged over the channel width, whereas in the saline clouds the velocity was measured along a straight line along the channel. This is the reason for the large variations in front velocity of the saline clouds which is a direct signature of the irregular nature of the front. We will see in $\S 6$ that the front velocity of avalanches measured along a path line also shows large fluctuations. Unfortunately, direct comparison between the front velocities of avalanches and of laboratory clouds is not possible because the filtering or averaging procedures used are different. 


\section{Analysis of the cloud velocities and conditions of sediment entrainment}

\subsection{Comparison of front velocity with (2.8)}

In figure 11 the variation with distance of the measured front velocities with and without sediment entrainment are compared with one another and with the values given by the model, e.g. by (2.8). There is a shift in the calculated velocities compared with the measured values. Especially in the case of the saline clouds the predicted velocities are about $15 \%$ to $20 \%$ larger. One possible reason for this is that the initial conditions do not correspond to the model conditions. When the gate is opened the fluid accelerates rapidly to a velocity of about $V_{0} \approx \sqrt{g h_{0}}$ which may be larger or smaller than the maximum velocity predicted by the model. In the case of the saline cloud $V_{0}<U_{m}$ and in the case of the suspension clouds $V_{0}>U_{m}$, because $h_{0}$ is larger. The slope angle modifies this initial velocity somewhat. In addition, during this acceleration time, the cloud changes its shape from the rectangular shape of the reservoir to the similarity shape. In the model, the shape is assumed to be half-elliptic. In fact, as has been mentioned before, there is a kind of wake behind the cloud. This wake induces a form drag that is neglected as a first approximation in the model. It would easily be possible to adjust the model to the experimental values by changing the initial conditions $\left(U_{0} \neq 0\right)$ and/or by changing the value of the added mass coefficient. Considering the very unsteady nature of the flow and the associated fluctuations in velocity and entrainment coefficient $E_{c}$, there is no reason to adjust the theoretical velocity values to the measured ones.

Of primary interest is the difference in front velocity of sediment-entraining and nonentraining clouds. The comparison between experimental results and the model from which the values of $\beta$ were determined was performed in the following way. The initial volume and the initial density are given quantities. The growth rate and the shape factors were measured independently. Thus, for non-entraining clouds, the velocity as a function of $x_{f}$ can be calculated directly from (2.8). For sediment-entraining clouds, the coefficient $\beta$ was adjusted such that the calculated velocity differences with respect to the non-entraining clouds corresponded to the measured velocity differences. This adjustment of the model to sediment-entraining cloud velocities gives values of $\beta \approx 2.5 \times 10^{-2}$ for the glass beads and $\beta h_{s}=0.2 \mathrm{~cm}(\beta=1)$ for the sawdust clouds. The value for the sawdust clouds is consistent with the observation that the whole layer was entrained into the flow.

\subsection{Sediment-entrainment capacity}

The procedure is to use the result $\beta h_{s}=0.2 \mathrm{~cm}$ obtained for the suspension cloud on a slope of $45^{\circ}$ to calculate $C_{m}$ from (2.23) and then $\gamma$ from (2.22). The value of $\gamma$ determined from these experiments can then be used to predict $\beta$ for the saline cloud and also for an avalanche (in $\S 6$ ). The suspension cloud is of interest here because the value of $\beta h_{s}=0.2 \mathrm{~cm}$ is obtained from direct observations and seems to be a limiting value for these conditions. The sawdust cloud with lower velocity or on a smaller slope did not entrain any sediment.

The mean concentration of the suspension cloud varies from about $7 \%$ at the start to $1.4 \%$ toward the end. At mid-distance downstream (about $1 \mathrm{~m}$ downstream) appropriate values for the two clouds are given in table 2.

From (2.23) (assuming equality) we obtain for the suspension cloud $\left(C_{m}-C\right) \approx 0.009$, hence $C_{m} \approx 0.03$ and (2.22) gives $\gamma=0.08$. For the saline cloud we find from (2.22) $C_{m} \approx 0.03$ and from $(2.23) \beta \leqslant 0.15$. This is larger but of the order of the experimental value $\left(\beta \approx 2.5 \times 10^{-2}\right)$. This suggests that the analysis is physically correct. If we were 


\begin{tabular}{lccc}
\hline & SC & DC & Avalanche \\
$C(\%)$ & 2 & 0 & $2-4$ \\
$U_{f}\left(\mathrm{~m} \mathrm{~s}^{-1}\right)$ & 0.11 & 0.3 & 53 \\
$H(\mathrm{~m})$ & 0.28 & 0.18 & 47 \\
$L(\mathrm{~m})$ & 0.7 & 0.59 & 254 \\
$\rho_{p} \times 10^{-3}\left(\mathrm{~kg} \mathrm{~m}^{-3}\right)$ & 1.06 & 2.5 & $0.4-0.9$ \\
$\rho_{s} \times 10^{-3}\left(\mathrm{~kg} \mathrm{~m}^{-3}\right)$ & 1.03 & 1.5 & 0.15 \\
$\rho \times 10^{-3}\left(\mathrm{~kg} \mathrm{~m}^{-3}\right)$ & 1.001 & 1.019 & 0.016 \\
$\theta(\operatorname{deg})$ & 45 & 32 & 25 \\
$w_{s} \cos \theta\left(\mathrm{cm} \mathrm{s}^{-1}\right)$ & 0.58 & 0.56 & $45-91$ \\
$K$ & 0.21 & 0.19 & 0.16 \\
$\alpha_{1}$ & 2 & 2 & 1.5 \\
$d(\mathrm{~mm})$ & 0.5 & 0.09 & $0.5-1$ \\
$h_{s}(\mathrm{~cm})$ & 0.2 & 2 & $100-150$ \\
$C_{f}$ & $10^{-2}$ & $2 \times 10^{-3}$ & $10^{-2}$ \\
$\left.u^{*}(\mathrm{~cm} \mathrm{~s})^{-1}\right)$ & 2.2 & 2.7 & 650 \\
$\operatorname{Re}_{*}$ & 11 & 2.4 & $325-650$ \\
$\Theta$ & 1.6 & 0.56 & $76-344$ \\
$\Theta_{C S}$ & 0.25 & $0.25 \dagger$ & 0.25 \\
$\dagger$ when $\operatorname{Re}_{T}=2$ & & &
\end{tabular}

TABLE 2. Characteristic values of the suspension (SC) and saline clouds (DC) and of the sediment layer. The values for the Swiss avalanche are included for comparison.

to follow the approach suggested by Fukushima \& Parker (1990), we would have obtained $\beta=0$ for the sawdust suspension cloud (it would only deposit sediment, which is not observed experimentally) and a value of $\beta$ more than 14 times smaller than the experimental value for the saline cloud.

As is seen in table 2, conditions are hydraulically smooth in the case of the glass beads and rough in the case of the sawdust layer. Consequently, the Shields parameter $\Theta$ is larger for the suspension cloud than for the saline cloud. For $\beta$ to be equal to the right-hand side of (2.23) it is necessary that $\Theta_{c s} / \Theta \ll 1$ which is nearly satisfied for the suspension cloud but not for the saline cloud. It is, therefore, not surprising that the experimental value of $\beta$ is less than 0.15 .

\section{Application to powder-snow avalanches}

\subsection{The Sion avalanche of 25 February 1999}

The avalanche for which fairly reliable data are available is one that was artificially triggered in Switzerland, in the Sion Valley, on 25 February 1999. Physically realistic values for this avalanche reported by Dufour et al. (2001) are: $A_{0}=200 \mathrm{~m}^{2}$, $\Delta \rho_{0}=150 \mathrm{~kg} \mathrm{~m}^{-3}, U_{f 0}=10 \mathrm{~m} \mathrm{~s}^{-1}, \Delta \rho_{s}=150 \mathrm{~kg} \mathrm{~m}^{-3}, h_{s}=1 \mathrm{~m}$. These initial values may vary from one avalanche to another but for large avalanches are in a similar range. $U_{f 0}$ is the initial front velocity related by $U_{f}=U\left(1+\frac{1}{2} \mathrm{~d} L / \mathrm{d} x\right)$ to $U_{0}$. It corresponds to the first point measured by Dufour et al. (2001). Because, initially, avalanches accelerate very rapidly, the position of this first point is very close to where the powder-snow avalanche was initiated. In the present case, the avalanche path can be approximated by an average slope angle of $25^{\circ}$. The width to height ratio of this 


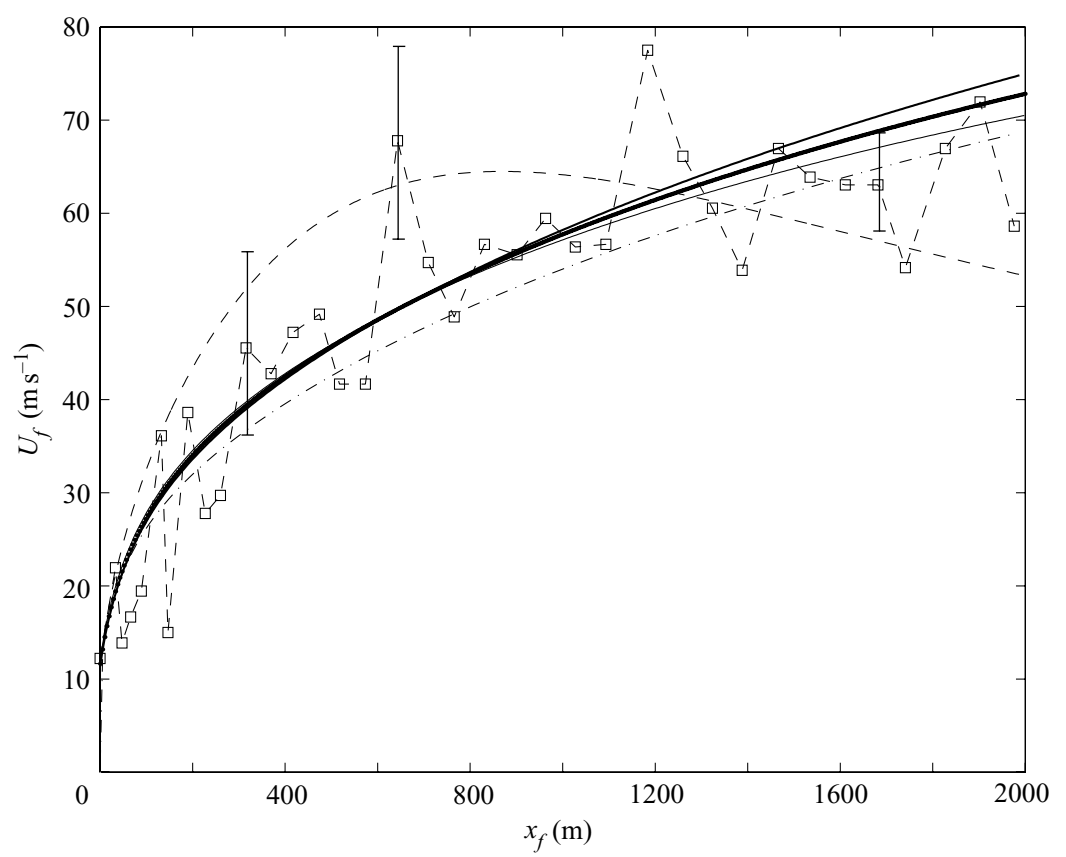

FIGURE 12. Front velocity $U_{f}=U\left(1+\frac{1}{2} \mathrm{~d} L / \mathrm{d} x\right)$ of an avalanche as a function of distance $x_{f}$ along the slope: $-\square---\square-$, measured values of Dufour et al. (2001); $\square$, model predictions for $\theta=25^{\circ}, E_{c}=0.25, \beta=1$ and $C_{f}=0 ;-\cdots$ - - model predictions when $\theta=25^{\circ}$, $E_{c}=0.25, \beta=0$ and $C_{f}=0$ (no snow entrainment); $\longrightarrow$, model predictions with $\theta=25^{\circ}$, $E_{c}=0.2, \beta=1$ and $C_{f}=0 ;-$, model predictions with $\theta=25^{\circ}, E_{c}=0.3, \beta=1$ and $C_{f}=0$; $-\cdot-\cdot-\cdot-$, model predictions with strong bottom friction $C_{f}=0.01, \theta=25^{\circ}, E_{c}=0.25, \beta=1$. Note that the values of $E_{c}$ used in these calculations are the corrected values $E_{c N B}$.

avalanche was about 3 to 5 and sometimes is confined laterally so that the assumption of two-dimensionality is acceptable (negligible lateral spreading). It has been reported by Dufour et al. (2001) that practically the whole snow cover was entrained along the avalanche trajectory, giving $\beta=1$. Dufour et al. (2001) determined the front velocity shown in figure 12 from images of the avalanche front taken with a video camera at $1 \mathrm{~s}$ time intervals. The velocity was calculated along a fixed trajectory, which is the reason for the relatively large velocity fluctuations about the mean. Indeed the front of an avalanche is very irregular (see figure 1). This is caused by large eddies which vary in time. As seen on figure 7 , the leading edge of laboratory gravity currents and clouds show a similar lobe-and-cleft structure also reported by Simpson (1997). Dufour et al. (2001) also performed velocity measurements by radar which gave access to the velocity inside the avalanche in addition to the front velocity; the velocity inside exceeded the front velocity by about $30 \%$ to $40 \%$. As was pointed out in $\S 2$ this result has important consequences on the flow structure and is responsible for the modification of the growth rate. The spatial growth rate and length of the avalanche was also estimated by Dufour et al. (2001) (private communications) from the geo-referenced photographs taken. At a distance of about $1500 \mathrm{~m}$, the height of the avalanche is in the range of 50 to $80 \mathrm{~m}$. The corresponding spatial growth rate is, therefore, on average, $\mathrm{d} H / \mathrm{d} x_{f} \approx 0.05$. The length has been estimated to be around $600 \mathrm{~m}$ at a distance of about again $1500 \mathrm{~m}$. 


\subsection{Comparison with the model}

In the present case, the origin chosen for $x_{f}$ corresponds to the initial state of the avalanche (first measurement point), e.g. for $U_{f 0}=10 \mathrm{~m} \mathrm{~s}^{-1}$. For the spatial growth rate we use the value determined from the laboratory Boussinesq clouds which gives for $25^{\circ}, \mathrm{d} H / \mathrm{d} x_{f B}=0.085$ and $k_{B}=3.3$. The corresponding air entrainment coefficient is $E_{c B}=0.32$. These values need to be corrected for inertial effects according to (2.17) and (2.19). Taking $\rho_{b} \approx 2 \rho \approx 20 \mathrm{~kg} \mathrm{~m}^{-3}$, the spatial growth rate is decreased by a factor of about 1.6 , giving $\mathrm{d} H_{N B} / \mathrm{d} x_{f} \approx 0.05$ for a slope angle of $25^{\circ}$. This spatial growth rate gives an avalanche height of about $80 \mathrm{~m}$ at a downstream distance of $1500 \mathrm{~m}$ and a length $L=5.4 H=450 \mathrm{~m}$. The entrainment coefficient according to (2.19) is $E_{c N B} \approx 0.25$.

The value of the saturation concentration can be determined from (2.22) using the experimental value $\gamma=0.08$ and the flow parameters halfway downstream $\left(x_{f}=800 \mathrm{~m}\right)$ given in table 2 . With these values we obtain from (2.22) $C_{m}=0.055$ to 0.25 and from (2.23) we obtain $\left(\Theta \gg \Theta_{C S}\right) \beta h_{s}=2.7$ to $3.6 \mathrm{~m}$. This means the snow entrainment is limited by the snow-layer thickness, i.e. the avalanche would be capable of entraining a snow layer of $3.6 \mathrm{~m}$ thickness. Although these are only rough values, it gives support to the observation that the avalanche entrains the whole snow cover. We, therefore, use $\beta=1$ in the model. However, if this kind of cloud moves onto nearly horizontal ground, rapid sedimentation would cause its arrest.

In figure 12 the model predictions are compared with the measurements by Dufour et al. (2001). The important point to notice is the difference of the front velocities with and without snow entrainment. Without entrainment a maximum is reached after the initial acceleration phase and then the velocity decreases in a way similar to Boussinesq clouds on inclines, as reported by Beghin et al. (1981). Substantial snow entrainment along the avalanche path is, therefore, required to ensure continuous acceleration to the large velocities observed. The model predictions are on the whole well within the measured values considering that for simplicity in the model the slope angle and hence $E_{c}$ were kept constant. In reality, this is not the case but the corrections by taking the respective local values remain small (second-order corrections). The bottom friction of the form shown in (2.1) lowers the velocity somewhat. The value of the coefficient $C_{f}$ is $C_{f}=10^{-2}$ because conditions are fully rough. A good fit of the experiments is obtained with this value.

\subsection{Comparison of the avalanche velocity with laboratory results}

\subsubsection{Evolution of the front velocities}

In order to compare laboratory results with avalanches, appropriate nondimensional variables have to be chosen. An appropriate velocity scale is $U_{m}$ which is the maximum velocity of non-entraining clouds (of the avalanche or the laboratory cloud). The corresponding length scale is the distance from the origin $x_{m}$ where this velocity maximum is reached. In figure 13 the front velocities $U_{f} / U_{m}$ are plotted as a function of $x_{f} / x_{m}$ for both the real and laboratory clouds. The characteristic velocity and length scales depend on the initial conditions and on the entrainment coefficient of ambient fluid only. The expressions for $U_{m}$ and $x_{m}$ are

$$
x_{m}=\sqrt{\frac{M}{3 N}},
$$




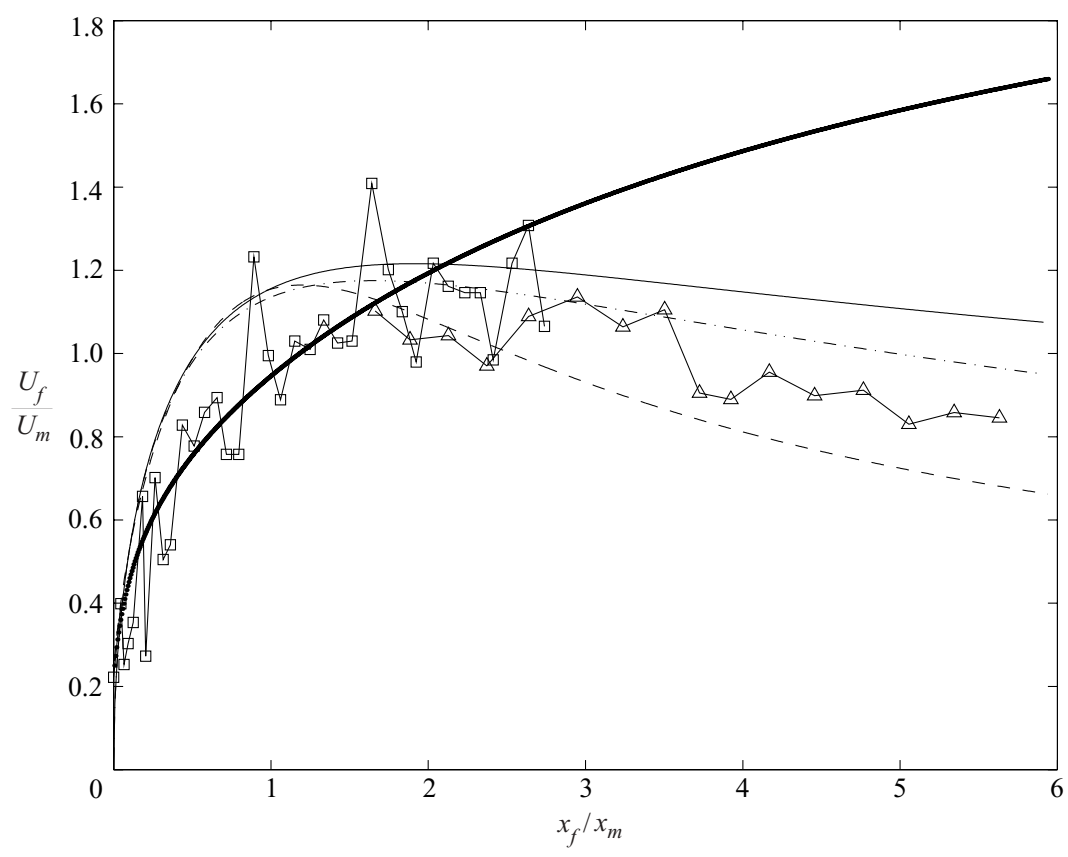

FIGURE 13. Non-dimensional avalanche and laboratory cloud velocities as a function of nondimensional distance: $-\square-\square-$, avalanche velocity measured by Dufour et al. (2001); , model predictions with $\theta=25^{\circ}, E_{c}=0.25, \beta=1$ and $C_{f}=0$; - - - - , model predictions when $\theta=25^{\circ}, E_{c}=0.25, \beta=0$ and $C_{f}=0$ (no snow entrainment); $-\cdot-\cdot-\cdot-$, calculated velocity for a laboratory cloud without sediment entrainment $\left(\theta=32^{\circ}, E_{c}=0.35\right)$; $-\triangle-$, measured front velocity of a laboratory cloud moving over a sediment layer; calculated velocity for the laboratory cloud with sediment entrainment with $\theta=32^{\circ}, E_{c}=0.35$, $\beta=1.2 \times 10^{-2}$ and $h_{s}=2 \mathrm{~cm}$.

$$
U_{m}=\frac{\sqrt{2 K M x_{m}+K B x_{m}^{2}+\frac{2}{3} K N x_{m}^{3}}}{M+B x_{m}+N x_{m}^{2}} .
$$

The values of $U_{m}$ and $x_{m}$ for the avalanche considered (Sion valley avalanche) are: $U_{m}=61.5 \mathrm{~m} \mathrm{~s}^{-1}$ and $x_{m}=945 \mathrm{~m}$ and for the laboratory saline cloud: $U_{m}=0.26 \mathrm{~m} \mathrm{~s}^{-1}$ and $x_{m}=0.41 \mathrm{~m}$.

The presentation of avalanche and laboratory cloud velocities in dimensionless variables shown in figure 13 is interesting because it shows more clearly the similarities and differences between the two. Without sediment entrainment the initial, acceleration phases, up to the velocity maximum, are practically identical. Beyond the maximum velocity the avalanche velocity decreases more rapidly than the laboratory (Boussinesq) cloud velocity because of the more rapid dilution of the large-density cloud (avalanche). Sediment entrainment reduces the initial acceleration in both cases. The reduction is, however, very weak in the case of the laboratory clouds because the sediment entrainment is very weak. The other important point demonstrated by figure 13 is that avalanches are limited mainly to the acceleration phase, even without snow entrainment where the maximum is reached only after a distance of about $1000 \mathrm{~m}$. On the other hand, laboratory data start almost where the avalanche stops. There is only a small overlap between the two. 


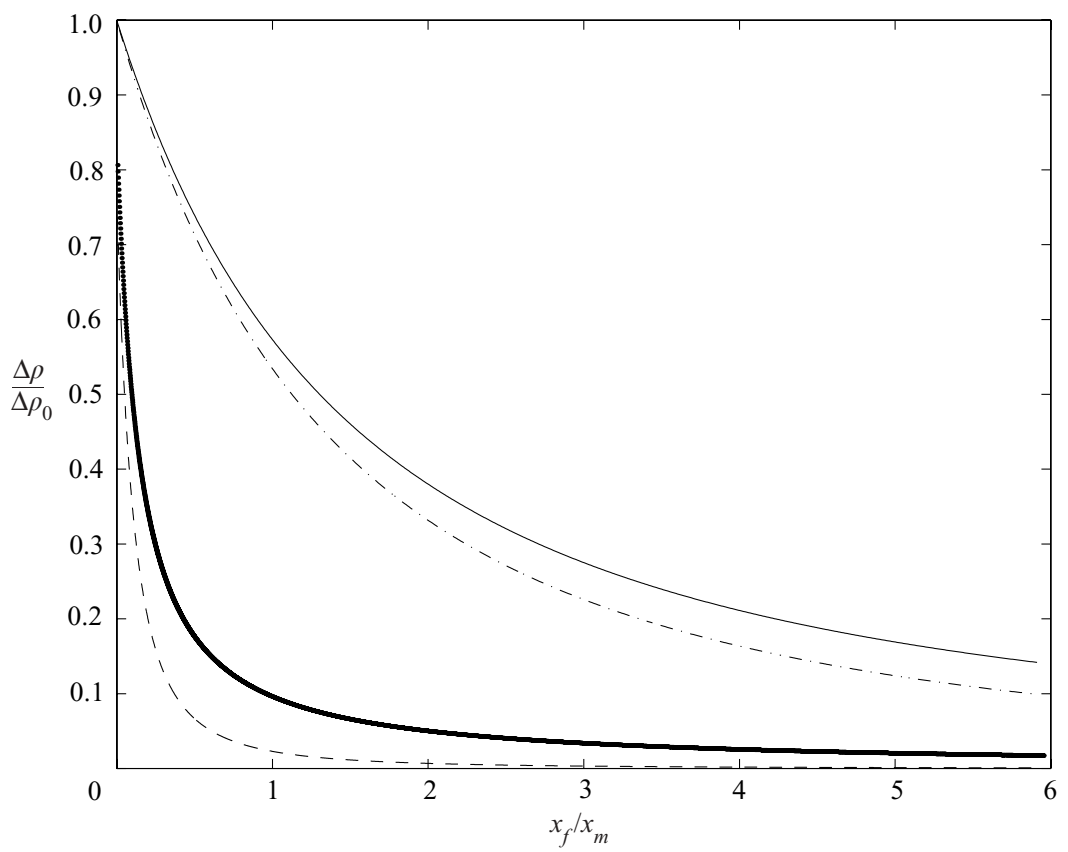

FiguRE 14. Comparison of density variations as a function of non-dimensional distance: - model predictions for an avalanche with $\theta=25^{\circ}, E_{c}=0.25, \beta=1$ and $C_{f}=0$; - - - model predictions for an avalanche when $\theta=25^{\circ}, E_{c}=0.25, \beta=0$ and $C_{f}=0$ (no snow entrainment); $-\cdot-\cdot-\cdot-$, calculated density variation for a laboratory cloud without sediment entrainment $\left(\theta=32^{\circ}, E_{c}=0.35\right) ;-$, calculated density variation for the laboratory cloud with sediment entrainment $\left(\theta=32^{\circ}, E_{c}=0.35, h_{s}=2 \mathrm{~cm}, \beta=2.5 \times 10^{-2}\right)$.

\subsection{Variation of density differences and overall Richardson number}

The variation in mean density difference, normalized by the initial density difference, as a function of dimensionless distance is plotted in figure 14. These values predicted by the cloud model should be close to the actual values because the model is in reasonably good agreement with the observed front velocities and the spatial growth rates. As expected, the mean density difference in the avalanche decreases much more rapidly than in the Boussinesq clouds. This is directly related to the large density difference between the ambient fluid (air) entrained and the avalanche density.

The overall Richardson number $R i=\Delta \rho g H \cos \theta / \rho U^{2}$ of the cloud, shown in figure 15 as a function of dimensionless distance, is also of some interest. By definition, $R i$ is initially infinite because $U=0$ and $H$ is finite and then falls off very rapidly to values less than 1 . The more rapid fall off of $R i$ in the avalanche case is related to the rapid decrease in density difference. The value of $R i$ in an avalanche is seen to be about half the value in a Boussinesq laboratory cloud. This could affect the air entrainment rate somewhat. However, what should be compared, if one wants to incorporate it in the ambient fluid entrainment coefficient, is the gradient Richardson number and these are likely to be closer to each other. Unfortunately, we do not have access to these values. 


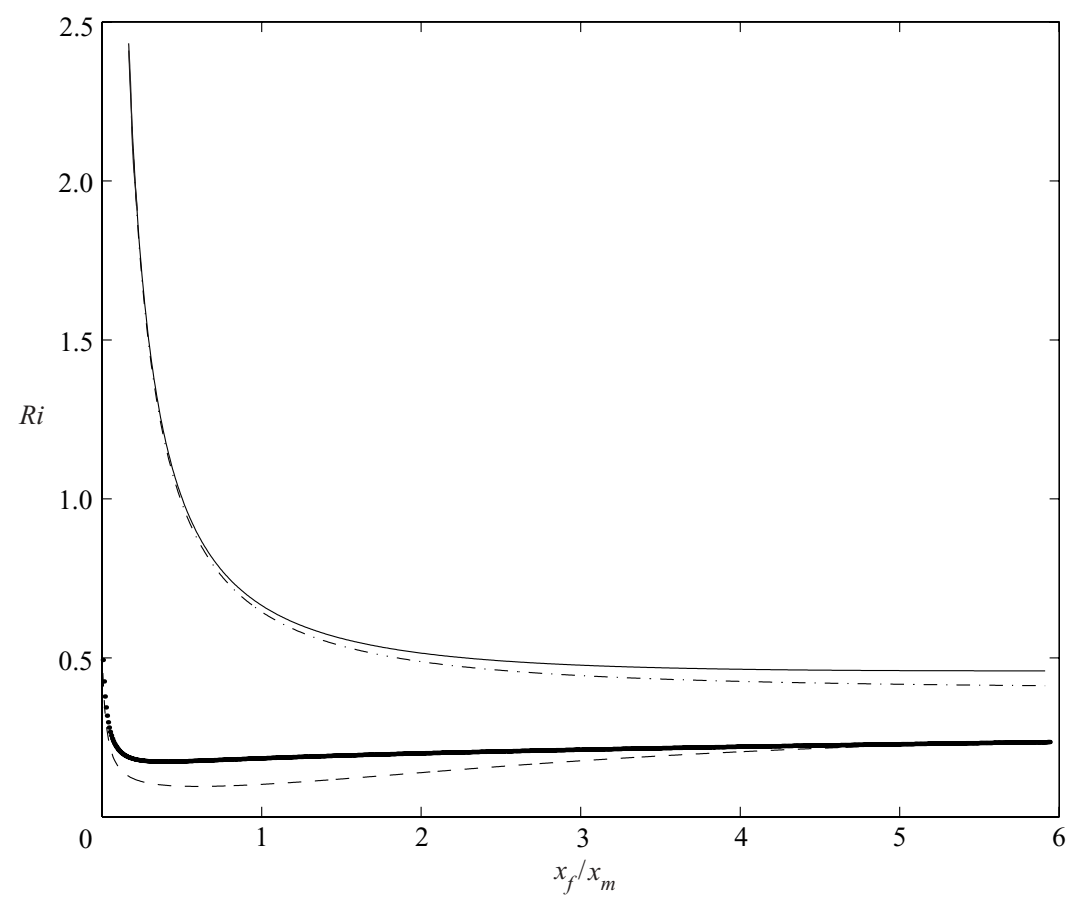

FIGURE 15. Variation of the overall Richardson number $R i=g \cos \theta\left(\left(\rho-\rho_{a}\right) / \rho\right) H / U^{2}$ as a function of dimensionless distance: - snow-entraining avalanche $\left(\theta=25^{\circ}, E_{c}=0.25\right.$, $\beta=1)$; - - - - - avalanche without snow entrainment $\left(\theta=25^{\circ}, E_{c}=0.25, \beta=0\right) ;-\cdot-\cdot-\cdot-$, non-entraining saline Boussinesq cloud $\left(\theta=32^{\circ}, E_{c}=0.35\right)$; $\longrightarrow$, sediment-entraining Boussinesq cloud $\left(\theta=32^{\circ}, E_{c}=0.35, h_{s}=2 \mathrm{~cm}, \beta=2.5 \times 10^{-2}\right)$.

\section{Conclusions and further discussion}

The cloud model developed indicates that avalanches, at least large powder-snow avalanches, are nearly always in an accelerating state. The analytical solution obtained for the front velocity is in agreement with recent measurement of Dufour et al. (2001). The model also shows the effect of snow entrainment along the avalanche path on the evolution of the front velocity. Besides the initial and topographical conditions, required for any model, the present model contains an air entrainment coefficient $E_{c}$, the spatial growth rate $\mathrm{d} H / \mathrm{d} x$ and a snow entrainment coefficient $\beta$ which need to be determined independently. In the Boussinesq limit, the former two are generic constants (within $10 \%$ to $20 \%$ variability) for a given slope angle and can be determined from laboratory experiments. The laboratory experiments presented in this paper were conducted in a flow regime close to the one of avalanches, that is in a range where the velocity is slowly varying around its maximum. The results obtained for the saline clouds are close to the ones obtained by Beghin et al. (1981) for their saline clouds. The growth rate for the suspension clouds is a little smaller. This is due to the settling of the particles. When these coefficients are used in an avalanche model an inertial correction has to be applied. An expression for this correction is proposed.

It is shown that in large avalanches the value of the entrainment coefficient is primarily determined (limited) by the properties of the sediment (snow) cover. When the snow cover depth $h_{s}$ is composed of a uniform (loose) powder-snow cover the 
coefficient $\beta=1$, meaning that the whole snow cover is incorporated. In laboratory conditions $\beta$ may vary from $\beta \ll 1$ to 1 depending on the sediment-layer thickness and on the type of sediment. In $\S 2$ an expression for $\beta$ in terms of the flow parameters and the sediment properties is derived and compared with laboratory experiments. The arguments given show that, generally, for avalanches snow entrainment is indeed limited by the thickness of the snow cover depth. When the avalanche moves onto nearly horizontal ground the front velocity decreases after some adjustment time and because of this, and also because of a change of the cloud structure, the maximum suspended load capacity decreases rapidly and sedimentation rather than entrainment occurs, bringing the avalanche to a stop. The effect of sediment entrainment on the front velocity is also shown in laboratory experiments and some indication of how sediment is entrained in a cloud is obtained from flow visualizations.

When the front velocities of the avalanche, considered together with the velocities of the laboratory cloud, are presented in dimensionless variables, the similarities of the two as well as the differences can be studied. In particular it is seen that avalanches occur mainly over downstream distances corresponding to the acceleration phase. This is true even without snow entrainment. Laboratory clouds, on the other hand, start almost where the avalanche stops. There is only a small overlap. The characteristic velocities and distances used in the non-dimensionalization comprise the initial conditions and the air entrainment coefficient.

One of the main aspects which would need to be substantiated in future work is the inertial effect on the cloud shape. Numerical simulation could be of great help in making some progress in this direction. Better controlled sediment entrainment experiments would also be most valuable.

This work was partially supported by a special grant (BQR) of the Institut National Polytechnique of Grenoble and by a fellowship of the 'Institut National des Sciences de l'Univers' under grant No. PNRN 00 CV 093. The authors wish to thank the CEMAGREF, Dept. ETNA for making the experimental facility available. Special thanks are due to Professor C. Ancey for helpful discussions during the work. Horacio Toniolo of the University of Minnesota provided the glass beads used in the experiments.

\section{REFERENCES}

Bagnold, R. 1962 Auto-suspension of transported sediment; turbidity currents. Proc. R. Soc. Lond. A $\mathbf{2 6 5}, 315-319$.

Baines, W. \& Hopfinger, E. 1984 Thermal with large density difference. Atmos. Environ. 18, 1051-1057.

Beghin, P. \& Brugnot, G. 1983 Contribution of theoretical and experimental results to powder snow avalanche dynamics. Cold Region Sci. Tech. 8, 67-73.

Beghin, P., Hopfinger, E. \& BritTer, R. 1981 Gravitational convection from instantaneous sources on inclined boundaries. J. Fluid Mech. 107, 407-422.

BRitTeR, R. \& Linden, P. 1980 The motion of the front of a gravity current travelling down an incline. J. Fluid Mech. 99, 531-543.

Brown, G. \& Roshko, A. 1974 Density effects and large structure in tubulent mixing layers. J. Fluid Mech. 23, 775-816.

Calder, E., Cole, P., Dade, W., Druitt, T., Hoblitt, R., Huppert, H., Ritchie, L., Sparks, R. \& YounG, S. 1999 Mobility of pyroclastic flows and surges at the soufriere hills volcano, montserrat. Geophys. Res. Lett. 26, 537-540.

Celik, I. \& Rodi, W. 1984 A deposition-entrainment model for suspended sediment transport. Tech. Rep. SFB 210/T/6. Sonderferschungsbereich 210, University of Karlsruhe. 
CELIK, I. \& RodI, W. 1991 Suspended sediment transport capacity for open channel flow. ASCE J. Hydraul. Engng 117, 191-204.

Dimotakis, P. 1986 Two dimensional shear layer entrainment. AIAA J. 24, 1791-1796.

Dufour, F., Gruber, U. \& Ammann, W. 2001 Avalanches: études effectuées dans la vallée de la sionne en 1999. Les Alpes 2, 9-15.

Ellison, T. \& Turner, J. 1959 Turbulent entrainment in stratified flow. J. Fluid Mech. 6, 423-448.

FukUShima, Y. \& PARKER, G. 1990 Numerical simulation of powder-snow avalanches. J. Glaciology 36, 229-237.

Graf, W. 1984 Hydraulics of Sediment Transport. Water Resources Pub. Littleton, Co.

Hadano, K. 1981 Study on the front of a turbidity current. Memoirs Faculty of Engng 41, 4. Kyushu University.

Hogg, A., Huppert, H. \& DAde, W. 1997 Erosion by planar turbulent wall jets. J. Fluid Mech. 338, 317-340.

Hopfinger, E.-J. 1983 Snow avalanche motion and related phenomena. Annu. Rev. Fluid Mech. 15, $47-76$.

Hopfinger, E. \& Tochon-Danguy, J.-C. 1977 A model study of powder snow avalanches. J. Glaciology 19, 343-356.

Hutter, K. 1996 Avalanche dynamics. In Hydrology of Disasters (ed. V. E. Singh), pp. 317-393. Kluwer.

Maslowe, S. \& Kelly, R. 1971 Inviscid instability of an unbounded heterogeneous shear layer. J. Fluid Mech. 48, 405-415.

Parker, G., Fukushima, Y. \& Pantin, H. 1986 Self-accelerating turbidity currents. J. Fluid Mech. 171, 145-181.

Simpson, J. E. 1997 Gravity Currents. Cambridge University Press.

Stacey, M. W. \& Bowen, A. J. 1988 The vertical structure of turbidity currents and a necessary condition for self-maintenance. J. Geophys. Res. 93, 3543-3553.

Strang, E. J. \& Fernando, H. J. S. 2001 Entrainment and mixing in stratified shear flows. J. Fluid Mech. 349-386.

Tochon-DanguY, J. \& Hopfinger, E. 1975 Simulation of the dynamics of powder snow avalanches. In Proc. Grindelwald Symp. on Snow Mechanics, vol. 114, pp. 369-380. IAHS-AIHS.

Tsang, G. 1971 Laboratory study of line thermals. Atmos. Environ. 4, 445-471.

TURner, J. 1972 Buoyancy Effects in Fluids. Cambridge University Press. 